\title{
PER UNA STORIA DEL NOTARIATO NELL'ITALIA \\ CENTRO SETTENTRIONALE TRA ASCESA E DECLINO. QUALCHE AGGIORNAMENTO*
}

\section{FOR A HISTORY OF NOTARIES IN CENTRAL NORTHERN ITALY BETWEEN RISE AND DECLINE. SOME UPDATES}

\author{
Maria Gigliola di Renzo Villata \\ Università degli Studi di Milano
}

\begin{abstract}
English: The aim is to trace a line of evolution of the notarial profession through the centuries from the late Middle Ages to the contemporary age in centralnorthern Italy between rise and decline, two poles of a multiform development. In the reconstruction, attention will be focused on some moments and junctions: the notary of the late medieval age intervenes in society and in institutions with a growing role, aware of his function and strong in his preparation, verified upon entering the business. The sixteenth century seems to be a moment of crisis, at least in the widespread perception in society. Preparation checks are intensified, archives are established, the 'nobility' of notaries is discussed. Between the end of the eighteenth and nineteenth centuries, state interventions multiplied up to the first unitary laws: only in 1913 a degree was required as a requirement for the profession.
\end{abstract}

Keywords: notary (history); requirements; notarial statutes

Abstract Italiano: L'obiettivo è quello di tracciare una linea di evoluzione della professione notarile attraverso i secoli dal basso medioevo all'età contemporanea nell'Italia centro-settentrionale tra ascesa e declino, due poli di uno sviluppo multiforme. Nella ricostruzione si concentrerà l'attenzione su alcuni momenti e snodi: il notaio dell'età bassomedievale interviene nella società e nelle istituzioni con un ruolo crescente, consapevole della propria funzione e forte della sua preparazione, verificata all'ingresso nell'attività. Il Cinquecento sembra rappresentare un momento di crisi, quanto meno nella percezione diffusa. Si intensificano i controlli della preparazione, si istituiscono archivi, si discute della 'nobiltà' dei notai. Tra fine Sette e Ottocento gli interventi statali si moltiplicano fino alle prime leggi unitarie: solo nel 1913 si chiederà, come requisito per la professione, la laurea.

* Italian Review of Legal History, 7 (2021), n. 16, pagg. 563-594

* https://riviste.unimi.it/index.php/irlh/index

* ISSN 2464-8914 - DOI 10.54103/2464-8914/16898. Articolo pubblicato sotto Licenza CC-BY.

* Il testo che segue, esito di ulteriori ricerche, approfondimenti e aggiornamenti, riproduce la struttura della lezione da me svolta al CERM il $1^{\circ}$ febbraio 2021; ha come base di partenza due miei saggi, il primo, di Renzo Villata, 2009a, con lo stesso titolo, il secondo, dedicato ai requisiti di accesso alla professione «tra cultura giuridica e pratica», di Renzo Villata, 2016. L'articolo non è stato sottoposto a procedimento di peer review. 
Parole chiave: notariato (storia); requisiti; statuti notai.

Sommario: 1. Una premessa. Tra ascesa e declino...: fortuna e sfortuna di una categoria professionale. - 2. Un'ascesa inarrestabile dopo tanto splendore? Ma volgiamoci al passato... I notai e la civiltà comunale. - 3. La formazione professionale: i requisiti per l'accesso alla professione a Bologna. -4 . II notaio e una moltitudine di competenze e attività: dal privato al pubblico. - 5. L'accentuarsi del controllo sull'attività notarile e gli archivi. Giambattista De Luca e i notai ne /l dottor volgare (1673)...: tra difetti e critiche ... verso la fine dell'ancien régime. -6 . Il notaio nell'Ottocento italiano: dalla legge del 25 ventoso anno XI ai progetti di riforma tardo-ottocenteschi del Regno d'Italia...: in dissolvenza. - 7. II notaio nel Novecento. -8 . Un bilancio attraverso i secoli.

\section{Una premessa. Tra ascesa e declino...: fortuna e sfortuna di una categoria professionale}

Appare necessario qualche brevissimo cenno, di ordine, per così dire, universale, per inquadrare la professione nel mondo di oggi, caratterizzato da una trasformazione, da un progresso (o, in determinati contesti, da una involuzione) di cui è indispensabile tener conto per meglio comprendere il suo evolversi nella società del XXI secolo, e... le sue lontane radici medievali che trovarono a Bologna un fertile terreno di sviluppo.

A livello globale II modello di notariato italiano, di tipo latino (civil law notaries), è presente in 87 Paesi del mondo, compresa Cina, Australia e Malaysia, e in 22 su 27 Paesi europei, copre oltre il 60\% della popolazione mondiale: si è espanso gradualmente negli anni. In questi paesi i notai sono giuristi di elevata formazione selezionati dallo Stato, per legge super partes e quindi in grado di offrire un'assistenza imparziale di cui sono garanti e responsabili.

Il public notary di tipo anglosassone è presente in Gran Bretagna, negli USA ed in altri Paesi; è responsabile solo dell'autenticità delle firme: il 'limite' della sua attività è di rendere talora opportuno per chi, ad esempio, compra e vende una casa, ricorrere alla consulenza di un avvocato, con maggiori possibilità di crescita del contenzioso.

Per quanto attiene all'Italia, La figura del notaio è descritta nell'art. 1, comma 1, della legge 16 febbraio 1913, n. 89: «I notari sono ufficiali pubblici istituiti per ricevere gli atti tra vivi e di ultima volontà, attribuire loro pubblica fede, conservarne il deposito, rilasciarne le copie, i certificati e gli estratti.». Nel seguito dell'articolo sono specificate ulteriori attribuzioni 'concesse'...

II notaio esercita un ruolo eminentemente documentale e certificativo, a tutela della pubblica fede, dell'esatta rappresentazione della volontà delle parti, della certezza di tutto quanto detto ed avvenuto in sua presenza, facendo piena prova di ciò che attesta negli atti: I'obiettività e neutralità è inerente all'ufficio, che esclude il perseguimento dell'interesse delle parti.

II requisito della laurea è infine introdotto, nel 1913, dopo che la presenza di 
una laurea negli aspiranti notai che si affacciavano alla professione aveva costituito una sorta di overeducation nel corso dell'Ottocento, soprattutto negli ultimi suoi decenni.

Ho voluto partire quasi dall'epilogo di una storia professionale, fortemente intrecciata alla competenza dei suoi appartenenti e alla capacità dimostrata, nel corso dei secoli, di affrontare le sfide che la società a loro contemporanea pone, perché la 'competenza', risultato di conoscenza e esperienza maturata sul campo, è, a mio avviso, una chiave di lettura per comprendere le ragioni di un'ascesa e di un declino della figura del notaio sul lungo periodo tra età medievale e moderna, fino alle vicende otto-novecentesche.

"L'imperizia dei notai distrugge il mondo", così scrive Wolfgang Unger a un'edizione, a cura di Andreas Perneder, della Summa Rolandina, uscita postuma a Ingolstadt nel 1544, poi ripresa in successive ristampe. Lo scopo è di tendere a una corretta, adeguata e aggiornata formazione tecnica dei notai di lingua tedesca attraverso una semplificazione della Summa Rolandina, non resa dunque nella sua interezza ma compendiata e resa più semplice per assicurarne una agevolata recezione nel mondo dei pratici di lingua tedesca.

Se ne sentiva allora la necessità nell'area germanofona: nel 1512 era stata emanata una Reichsnotariatsordnung da Massimiliano I per porre rimedio ad alcuni 'mali' degli appartenenti alla categoria, giudicati «defectuosi et indigni» (versione latina), ignoranti e di scarsa moralità anche perché condannati per falso o per altri crimini, diffamati pubblicamente, in una 'impietosa' fotografia delle carenze di uomini che avrebbero dovuto eccellere per qualità. Occorreva dunque assicurare una migliore preparazione, fondata sulla teoria, integrata dalla opportuna conoscenza del diritto comune e delle consuetudini locali (in una parola essere iurisperiti), oltre che sulla pratica, e operare in modo che i soggetti così preparati potessero svolgere le loro funzioni «legaliter, fideliter et sincere» ${ }^{1}$.

Da subito, nel filo della narrazione che si svilupperà in queste pagine, dal taglio inevitabile storico-giuridico data la mia 'specializzazione', compare la Summa Rolandina, che è, per così dire, il fiore all'occhiello di una produzione pratico-scientifica nell'ambito notarile proveniente da un notaio, Rolandino de' Passeggeri, personaggio eminente nella società bolognese tra la prima e la seconda metà del XIII secolo²: sarà determinante nel creare una sorta di personaggio 'mitico', all'origine di una rappresentazione di una categoria professionale, di cui testimonia al meglio le 'virtù', al di là delle difficoltà personali conosciute nel corso dell'ascesa politica. Dunque uomo di cultura, impegnato anche sul versante didattico, e politico di notevole peso nella vita bolognese del Duecento.

\footnotetext{
${ }^{1}$ Schmoeckel, 2012, pp. 29-74.

${ }^{2} \mathrm{Si}$ cita qui un'edizione ampiamente conosciuta come quella di Rolandino de' Passeggeri, 1546/1977, ma, come si è accennato, la tradizione manoscritta e a stampa dell'opera è estremamente ricca: cfr. sull'opera nelle sue diverse sfaccettature i saggi raccolti in Tamba (ed.), 2002.
} 
Sono connotazioni non rare nel notaio dell'età comunale (e anche oltre), che svolge una funzione di spicco nella società del tempo. Ma la sua 'immagine' sembra volgere al declino, forse - azzardo, come succede non solo nell'attività notarile - a causa dell'incancrenirsi di certe abitudini che possono apparire quasi connaturate alle peculiarità della professione: oltre ad una sua, assolutamente non scontata, propensione a agire 'scorrettamente', per non usare termini più pesanti, interagisce infatti con una clientela privata (e anche pubblica... ma, in questo caso, forse - si spera - meno propensa alla falsificazione) di cui deve soddisfare gli interessi patrimoniali, a volte non coincidenti con l'interesse primario della veridicità della documentazione autenticata.

Il predicatore bagnacavallese Tommaso Garzoni, eclettico autore de La Piazza universale di tutte le professioni del mondo, una sorta di bestseller all'epoca, pubblicata per la prima volta a Venezia nel 1585 e oggetto di attenzione anche al di fuori dei nostri confini, tra Spagna e Germania, ove, nel Seicento, fu ripubblicata più volte, delinea con fine arguzia alcuni tratti della professione notarile lasciando al lettore un'impressione fortemente negativa degli esiti della sua attività, almeno all'epoca del Garzoni, riflesso di una cattiva fama guadagnata nel tempo.

Giudicato variamente da chi, storico, s'è occupato di lui e della sua opera, ora conformista, ora spregiudicato, in ogni caso, a mio avviso, attento osservatore della realtà a lui coeva, dipinge certo del notaio un ritratto non lusinghiero, espressione, per citare ancora chi ha studiato in profondità il suo pensiero, «delle paure collettive di una società che si sentiva minacciata nel suo ideale di stabilità di fronte all'insicurezza... $\nu^{3}$. La lettura delle sue notazioni in proposito mi sembra significativa per delineare alcune caratteristiche de' Nodari attraverso la sapida raffigurazione, anche plasticamente efficace, forse un po' forzata, del loro operare offerta dall'Autore, che non si risparmia nell'attribuire loro tutti i peggiori comportamenti, dall'ignoranza alla intenzionale mala fede, ai confini di una corretta morale e sana coscienza

L' ufficio, overo la profesione de ' Nodari è cosa degna, \& honorevole in se stessa, come si trahe dal Codice...; perché il Nodaro è persona publica, \& l'ufficio del Giudice senza la persona sua non può commodamente esercitarsi . Eglino son fra leggisti ( come dicono Giovanni de Platea , \& Orlandino nella sua somma ) con più vocaboli addimandati, cioè Notarii dal notare che fanno le civili attioni : Tabelliones, perché anticamente si soleva scrivere in certe tavole di legno: Scrineari, perchè gli instromenti scritti da loro son soliti à riporsi dentro ne 'scrigni : Librarii, perché l'ufficio loro è di librare , \& pesare i negocij, che passan per le lor mani , giustamente, fedelmente: scribi dallo scriver ch'essi fanno con quella pennaruola sempre a canto, che somiglia alla tasta d'un Cirugico... Hanno poi questi nodari la camiscia imbrattata ancora loro molto bene, perché [...] alle volte tratti dall'ignoranza fanno instromenti inetti, et confusi, o difettuosi, et invalidi, perché non hanno le debite solennità, laonde restano le persone dannificate, et ne suscitano

\footnotetext{
${ }^{3}$ Gnavi, 1990, spec. p. 71. V. Niccoli, 1999.
} 
litigii d'importanza con manifesta ruina delle parti. Alle volte anco scientemente, et a posta fanno instromenti falsi, come ne' contratti di compre, o pagamenti, ovvero ne' testamenti, intricando le lor conscienze nel falso; et seco i testimonii insieme, quai pigliano a lor modo, per buscar qualche imboccata da persone malvagie, et senza un'oncia di conscienza al mondo. Et qualche volta occultano le scritture giovevoli, et necessarie agli altri; a instanza di qualcuno, per giovare a quello, et nuocere al resto; come i legati delle pie cause passano sovente per questo trabocchello. Oltra di ciò vedranno talhora, che un contratto sarà violato et fatto per via d'estorsione, come nelle rinoncie, che fanno alcuni ch'entrano nelle religioni, o ne' contratti di matrimonii sforzati, o ne' testamenti di quelli che testano, havendo perso l'uso di ragione, e non potendo legittimamente testare; e nondimeno, pur che trovino da colare, come becchi et castroni colano all'herba, et si rogano via senza risguardo alcuno, et senza alcuna consideratione; né meno si fan pregar talvolta a formare uno instromento usurario, come si usa in molti luoghi della Graffignana, per graffiar bezzi a tutte le foggie, i quali corrono per mezzo delle linee scritte, potendovi passar fino ai Zanfroni, tanto le fanno larghe, et l'una dall'altra distante, per guadagnare $[. . .]^{4}$.

La falsificazione è reputata una 'contingenza' verificabile in una serie di atti di cui la storia abbonda ${ }^{5}$ dai testamenti ${ }^{6}$ e, in particolare, ai legati pii, ai contratti di vario contenuto, alle rinunce alla successione ${ }^{7}$, fatte, soprattutto da donne, in occasione del loro matrimonio e della monacazione più o meno forzata, ma pure da uomini al momento del loro ingresso in un ordine religioso... Non solo Garzoni, in pieno Cinquecento, stigmatizza questo 'malcostume' (per non infierire sulla gravità del comportamento), ma pure Giovanni Boccaccio, che ce ne trasmette, nell'efficacissimo ironico racconto su Ser Ciappelletto, della prima novella del suo Decameron, un'immagine, addirittura di santificato dopo avere purgato i suoi peccati con una confessione ${ }^{8}$.

Dall'altro capo della 'barricata' ci sono i notai che difendono la professione elogiandone le virtù e 'nobilitando' la loro attività. Uno di questi è Antonio Giustiniano Roccatagliata che, a metà del Cinquecento, scrive un elogio, in forma di dialogo, intessuto di lodi per la nobilissima ars notariorum, tuttavia 'velato' e sminuito da una constatazione, alla quale si deve dar credito, sulla pochezza della categoria, fatte salve le eccezioni: «quia notarii in urbe nostra (bonis semper exceptis) viles sunt et ignavi, indocti et arrogantes et paene stulti miserrimique»,

\footnotetext{
${ }^{4}$ Garzoni, 1996, pp. 171-172.

${ }^{5}$ Cfr. Murray - Dolezalek, 2020, pp. 113-230.

${ }^{6} \mathrm{Mi}$ sia consentito di rinviare, per uno sguardo sui testamenti, istituto veramente nevralgico nell'attività notarile, a di Renzo Villata, 2009b.

${ }^{7}$ A partire dai saggi di Meynial, 1900-1901, pp. 108-142 (1900) e pp. 241-277 (1901). V. anche Schmidt, 1988, pp. 691-711; Preto, 2020, passim, spec. pp. 58-61, 84-87; v. anche Varanini, 2006, pp. 1-27 (http://www.rmojs.unina.it/index.php/rm/article/view/urn\%3Anbn\%3Ait\%3Aunina-3190).

${ }^{8}$ Cfr. Boccaccio, 2017.
} 
vizi questi da attribuire ai singoli, non all'ars baluardo della fides ${ }^{9}$. Contribuiscono all'esaltazione, per dare solo alcuni nomi di autori che danno alle stampe il loro 'panegirico', Giovanni Magoni, causidico pavese autore di Lucernae moralis partes duae. Prima de authoritate notariatus... secunda de notariatus fidelitate, addirittura riedito tra il 1602 e il 1611, il giureconsulto milanese Francesco Osio con il De antiqua tabellionum nobilitate... (1636), Placido Puccinelli, che scrive in volgare, a metà del Seicento, Della fede e nobiltà del notaio ${ }^{10}$.

Un altro, il notaio fiorentino Ser Matteo Bruneschi, prima provvisto di una clientela privata, poi notaio-attuario nei vari Tribunali centrali fiorentini, compone, nella copia manoscritta conservata nell'Archivio di Stato di Pisa, datata al 1621, una Apologia... nella quale si difende la honorata professione de' notari procuratori et causidici dai falsi calunniatori.

Ser Matteo non comprende le ragioni delle critiche malevole e si attiva «per non lasciare cattive impressioni nell'animo di chi sente, et per non lasciar indifese le cose, che più difender si debbono... non è però che non faccia mestiere difendere quelle cose che talora sono biasimate a torto, et inalzar quelle, che in manco expectatione, che sia conveniente, si tengono "; giudica il ceto composto da "uomini... di più sottile ingegno et di più perfetto giudizio ed intelligenza che molti altri»: una sorta di modello per i trattati sulla 'nobiltà' del ceto notarile, fondati sulle 'provanze di nobiltà'(Montorzi) $)^{11}$.

Altri si succedono nell'esercizio teso alla difesa del notariato: Montorzi citava, in particolare, Matteo Neroni, autore di un inedito Nobiltà del tabellionato, Virginio Scolari già Colombani, autore di una Scrittura poi pubblicata dal Puccinelli, e Agostino Coltellini, a cui si deve una Instruzione $a^{\prime}$ novizi per matricolarsi notai...12.

Ancora, più di un secolo dopo, è la volta di Ludovico Antonio Muratori che, ne $I$ difetti della giurisprudenza (1742), mette sul banco degli imputati i 'poveri' notai', assieme ad altri uomini di legge: per i Nostri addita la difficoltà di «...scoprire ed interpretare la volontà ed intenzione degli uomini, con ricavarla da i fatti, o pur dalle parole degli stessi mortali», aggiungendo:

"Dirò di più: non han poca colpa in ciò alle volte gl'ignoranti Notai, che o non intendono la mente de' contraenti e testatori; o se l'intendono, l'esprimono così trascuratamente o confusamente, che resta fondamento a due contrarj Avvocati di spacciarla e pretenderla cadauno favorevole al proprio Cliente. Allorché certo

\footnotetext{
${ }^{9}$ Roccatagliata, (1556), p.n.n.; Sinisi, 1997, p. 173.

${ }^{10}$ V. di Renzo, 2009a, pp. 45-46. Puccinelli, s.d. (ma i riferimenti interni conducono a una data a metà del secolo).

${ }_{11}$ Montorzi 1985, spec. p. 21, 36, 38 ss., con il proemio dell'Apologia di Matteo Bruneschi Notaio fiorentino, nella quale si difende la honorata professione ( $r$ ) de' notari procuratori et causidici dai falsi calunniatori (pp. 38-42).

${ }^{12}$ Ivi, pp. 48-59.
} 
Dottore della nostra Città osservava Notai, che stendevano qualche contratto, o ultima volontà delle persone, solea dire sorridendo a' suoi colleghi: mirate colui: egli ora sta lavorando per me» ${ }^{13}$.

Dunque ignoranza, negligenza, incapacità di esprimersi in un linguaggio chiaro, non involuto aggiungerei, sì da escludere fraintendimenti e interpretazioni contrapposte, che avvocati pronti ai cavilli possono sfruttare a vantaggio dei loro clienti. La 'battaglia' preilluministica contro la giurisprudenza si esprime già con tutte le armi a disposizione prendendo come favoriti bersagli gli esponenti del ceto forense, nella vasta gamma delle sue articolazioni.

L'epoca d'ora della 'Repubblica dei notai' (Cencetti) ${ }^{14}$, per indicare il forte potere nelle istituzioni politiche e sociali bolognesi di allora (a metà del XIII secolo) nonché la sua azione 'pervasiva' a tutto campo, sembra decisamente al tramonto... ; i secoli dal Cinque al Settecento rivelano le molte difficoltà della categoria professionale; l'Ottocento vedrà, almeno in Italia, un percorso, in mezzo a molte 'asperità', finchè, nel Novecento, la professione registrerà una progressiva ascesa, fino alle difficoltà del XXI secolo.

Una storia, quella del notariato, in cui i momenti di ascesa si alternano a quelli di declino, si intersecano al vissuto delle istituzioni in cui svolgono ruoli spesso imprescindibili e, perciò, sono oggetto di molteplici attenzioni da parte della comunità politica e della società dei privati.

\section{Un'ascesa inarrestabile dopo tanto splendore? Ma volgiamoci al passa- to... I notai e la civiltà comunale}

L'emergere del ruolo della figura del notaio, già sviluppata in età altomedievale ${ }^{15}$, è intrecciata allo sviluppo della civiltà comunale, alla diffusione della scrittura e al suo utilizzo per uno scopo di certezza del diritto.

II notaio svolge attività al servizio dei privati e della pubblica autorità: opera nell'ambito privatistico, con la redazione di documenti per la clientela privata, dotati di pubblica fede ${ }^{16}$, che assicurano certezza del diritto; collabora con le istituzioni nella redazione dei verbali dell'attività istituzionale, che acquistano un crisma di legittimità anche grazie alla scrittura da parte del notaio stesso, con variato grado di funzioni, fino all'attività giudiziaria, con la presenza del notarius maleficiorum, del notaio delle cause civili ${ }^{17}$. I notai documentano su questo versante l'attività svolta in loro presenza e svolgono funzioni 'più incisive', ora anche da giudice 'decisore' di controversie, spesso con funzioni di volontaria giu-

\footnotetext{
${ }^{13}$ Cfr. Muratori, 1742, pp. 12-13. V. di Renzo Villata, 2002b, pp. 83-119, spec. pp. 99-100.

${ }^{14}$ Cencetti, 1977. V. ora Grillo, 2017, pp. 99-114.

${ }^{15}$ Cfr. Amelotti, Costamagna, 1995; Cammarosano, 2001, spec. pp. 115-116, 142, 148-150, 153, 163, 168, 182, 190, 280; Condorelli, 2014.

${ }^{16}$ Montorzi, 2004, passim, ora Tarantino, 2010.

${ }^{17}$ V. Sinisi, 2006; Sinisi, 2014, Sinisi, 2020.
} 
risdizione (nomina di tutori, emancipazione di minori ecc.). Nell'ambito delle loro competenze v'è anche il ruolo nella funzione legislativa: il notaio è parte attiva nella composizione degli statuti e delle altre manifestazioni normative. Si trova coinvolto pure nelle complesse procedure per l'autenticazione dei miracoli e per la beatificazione ${ }^{18}$.

Come ha rilevato, ormai due decenni fa, Gian Giacomo Fissore, "Una delle caratteristiche della civiltà comunale è certamente rappresentata dall'espansione dell'impiego della documentazione scritta in ambito cittadino, con una pervasività della strumentazione notarile che sembra investire tutti i momenti della vita $\mathrm{d}^{\prime}$ interrelazione con una minuziosità quasi maniacale $»^{19}$.

Nel processo di trasformazione epocale della società coeva i notai hanno un posto di assoluto rilievo, rafforzato nel tempo, diversamente da quanto accade in altri paesi europei con differente tradizione, come, per fare un esempio tra $i$ tanti possibili, nell'Inghilterra del XIII secolo.

Giovanni da Bologna, autore della Summa de his quae in foro ecclesiastico coram quibuscunque iudicibus occurrunt notariis conscribenda, scriveva, al tramonto del Duecento, dopo avere esercitato l'attività notarile in area britannica: «Gli Italiani, uomini prudenti, vogliono avere uno strumento pubblico praticamente per ogni contratto che stipulano; gli Inglesi sono invece proprio all'opposto e uno strumento viene richiesto solo quando è essenziale ${ }^{20}$.

Da Bologna ${ }^{21}$ a Parma ${ }^{22}$ e Ravenna ${ }^{23}$, da Genova ${ }^{24}$ a Milano ${ }^{25}$, a Cremona ${ }^{26}$, da

${ }^{18} \mathrm{Cfr}$. i saggi raccolti in Michetti (ed.), 2004.

${ }^{19}$ Fissore, 1998, pp. 39-60, spec. p. 39.

${ }^{20}$ V. Cheney, Stein, Brooks, Helmholz, 1972; Cheney, 1991 (sui Notai pubblici in Inghilterra, spec. p. 178); anche Clanchy, 1979, p. 37; più di recente Ramsay, 2009 (a pp. 390-391 la più recente storiografia).

${ }^{21}$ La bibliografia è ricchissima: a titolo meramente esemplificativo v. i saggi raccolti in Cencetti, 1977, pp. 217-352; Tamba, 1988; Tamba, 1998.

${ }^{22}$ Cfr. A. Aliani, 1995.

${ }^{23}$ V. De Lorenzi, 1961-1962.

${ }^{24}$ II notariato genovese e ligure è stato oggetto di molteplici attenzioni dal secolo scorso. V., ad es., Vitale, 1949; Costamagna,1970; più di recente Sinisi, 1997; Rovere, 2013; già Rovere, 2009; Ruzzin, 2015; Ruzzin (ed.), 2018.

${ }^{25}$ Cfr. ad es. Liva, 1979. Le edizioni critiche di atti rogati a Milano tra il secolo XII e XIII sono numerose.

${ }^{26}$ Cfr. Leoni, 2013. 
Torino ${ }^{27}$ ad Asti ${ }^{28}$ e a Novara ${ }^{29}$, a Vercelli ${ }^{30}$ e ad Alessandria ${ }^{31}$, da Venezia ${ }^{32}$ a Verona $^{33}$, da Padova ${ }^{34}$ a Treviso ${ }^{35}$, a Belluno ${ }^{36}$, da Firenze ${ }^{37}$ a Prato ${ }^{38}$ e Pisa ${ }^{39}$, da Siena ${ }^{40}$ a Lucca $^{41}$ e ad Arezzo ${ }^{42}$, per non parlare dell'Italia meridionale ${ }^{43}$, ogni centro comunale di importanza più o meno grande, portatore di una sua storia attraverso $i$ secoli dal medioevo all'età moderna sino alla contemporanea, si presta a divenire un laboratorio privilegiato per una storia del locale notariato, che ne 'registra' insieme le vicende.

\section{La formazione professionale: i requisiti per l'accesso alla professione a Bologna}

La professione del notaio, sempre più nevralgica, richiede una formazione: per citare le figure più rilevanti e più conosciute per il loro contributo allo sviluppo di una 'metodologia' applicata attraverso istruzioni e modelli da seguire nella pratica professionale spiccano i nomi di Ranieri da Perugia, Salatiele e, ovviamente, di Rolandino de' Passeggeri.

Prendiamo le mosse da Ranieri da Perugia (ca. 1190-1255) che, tra il 1214 e il 1216, completa il Liber formularius, un testo per la scuola e la pratica notarile; apre una scuola di notariato; tra il 1226 e il 1233 compone l'Ars notariae, divisa in tre parti, dedicate rispettivamente a contratti, atti giudiziari, disposizioni di ultima volontà; nel 1219 ca. coordina il Registro grosso, dai documenti raccolti nell'archivio del Comune, quelli di preminente interesse, specie per la gestione dei beni pubblici. Ho riassunto in pochi tratti il respiro di una vita che segna, nel-

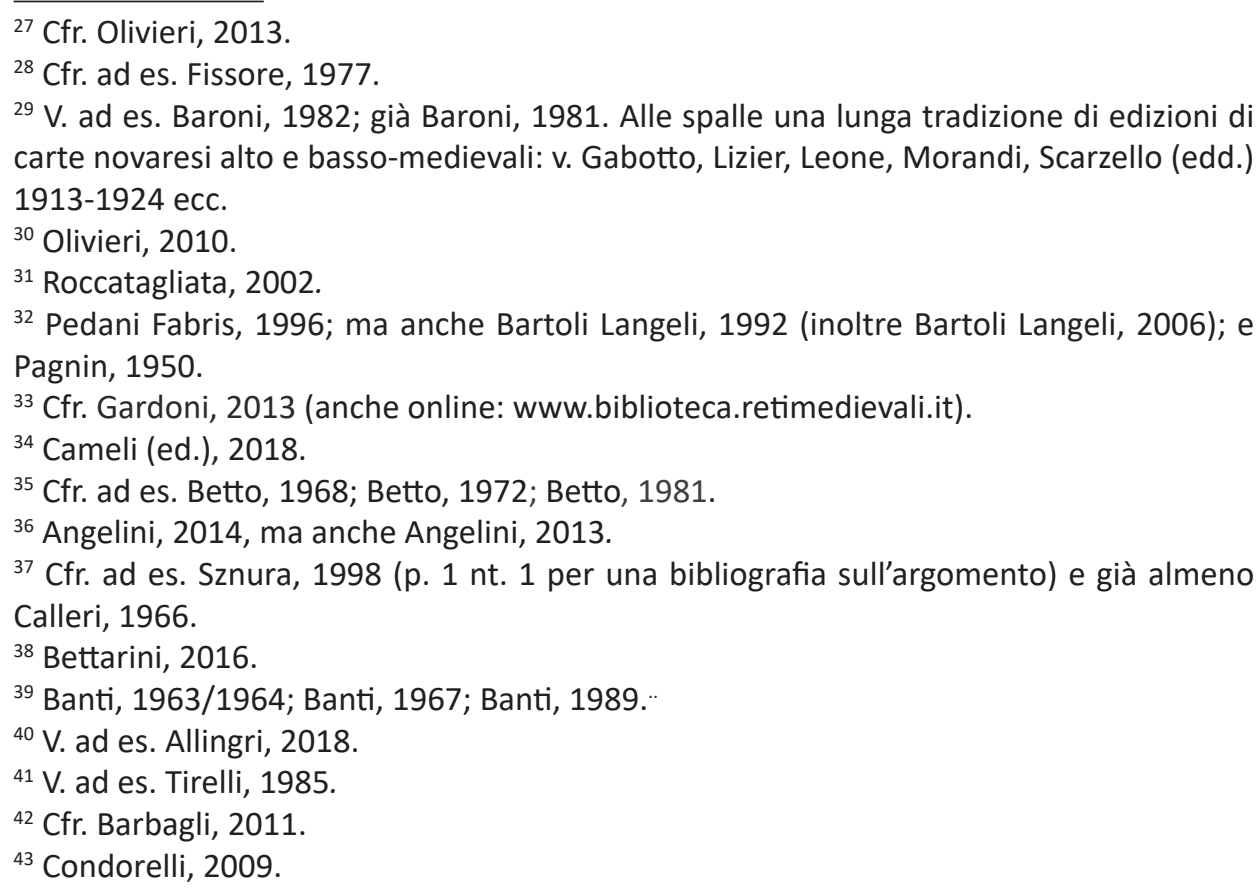


la storia del notariato, un momento di snodo di grande influenza, forse, anzi di certo di minore rilievo rispetto ad altri che seguiranno, eppure nevralgico nell'indicare una direzione di marcia.

Un altro personaggio che merita di essere menzionato, in questa inevitabilmente sommaria carrellata, è Salatiele, che apre una scuola di notariato a Bologna e manifesta propositi per così dire bellicosi verso la categoria dei notai: vuole alzarne il livello culturale e le sue opere stanno a dimostrare le sue intenzioni. l'Ars notarie (1242-1243), a cui segue la Summula de libellis, scritta a partire dal 1245 e edita nei Tractatus universi iuris nel 1584, poi riedita in tempi più vicini a noi, non priva di spunti polemici, ripresi in seguito da Rolandino ${ }^{44}$, rappresenta un importante passo verso la costruzione di una professione notarile, prima di lui fondata su una vivace, ma indubbiamente elementare tradizione di formulari pratici, e, grazie alle sue opere e a quelle, indubbiamente più conosciute, di Rolandino, innalzata alla dignità giuridica, "accolta nel cielo del diritto romano» ${ }^{45}$. L'opera, che tratta largamente di famiglia, successioni, contratti, di processo civile, di compromesso e arbitrati ${ }^{46}$, di processo penale ... e di un universo giuridico bisognoso di certezza, oltre che dell'intervento 'salvifico' del notaio, è accompagnata da glosse, ordinate, destinate a spiegare passo passo la successione dei capitula e a fornire uno strumentario utile a meglio penetrare la sostanza giuridica delle formule e ad agevolarne una piena comprensione, perciò «una sorta di manuale stringato, ma aggiornato» (Birocchi ${ }^{47}$ ), utile per l'insegnamento. Ripresa, in tutto e in parte, in vari brani, in un'anonima Ars notariatus tra Quattro e Cinquecento, non fu opera che godette di una gran fortuna, 'eclissata' dall'astro più splendente di Rolandino. Salatiele è notaio colto, cita Cicerone, Virgilio, Orazio, Ovidio, Aristotele, Boezio, Agostino, insegna nella sua scuola di notariato, svolge attività presso la pubblica autorità, tanto sul piano tecnico, quanto su quello diplomatico e politico, fino ad essere bandito dalla città di Bologna ${ }^{48}$.

E poi doveroso è rammentare la 'fulgida stella' di Rolandino con la sua Summa artis notariae, "onnivoro maestro assunto a fondatore dell'ars» ${ }^{49}$ : nel suo dominare teoria e pratica, 'riesce' a diffondere la sua concezione e si giustifica il successo che merita attraverso una diffusione capillare attestata da più di 200

\footnotetext{
${ }^{44}$ In TUI, III/2, ff. 79vb-89vb; ora anche in ed. moderna a cura di A. Grazia, 1972. V. Orlandelli, 1989.

${ }^{45}$ In questi termini Giansante, 2017, si ricollega a Orlandelli, 1961, p. 507.

${ }^{46}$ V. di Renzo Villata, 2002a; Chiodi, 2002; Massetto, 2002; Padoa Schioppa, 2002; Storti Storchi, 2002.

${ }^{47}$ Birocchi, 2013b.

${ }^{48}$ Cfr. Birocchi, 2013b; Giansante, 2017 (già, con riguardo a due peculiari aspetti del notaio operante nella città felsinea, Giansante, 2000, ma anche Sinisi, 2002, spec. pp. 175176; Bruschi, 2006 (a.i)).

${ }^{49}$ Cfr. Ferrara, 1983, p. XLI; v. come primo approccio, la bella voce di Birocchi, 2013a; anche Condorelli, 2014.
} 
manoscritti, conservati in Europa, 55 nell'area tedesca ${ }^{50}$. Si attua, attraverso questa riuscitissima opera, una "trasfusione equilibrata di una prospettiva tendenzialmente scientifica nella pratica notarile, per così dire una sua 'scientificizzazione' nell'armonioso incontro di istanze pratiche e teoriche sempre da aggiornare, le prime ispiratrici di modelli negoziali ben congegnati, ricchi di clausole frutto di un panorama culturale dotto e aggiornato, le seconde, espresse nel corredo interpretativo (Aurora) ${ }^{51}$, felice prospetto ordinato degli esiti raggiunti da un lavorio dottrinale svoltosi intorno a certi temi sul lungo periodo e bisognoso di un continuo adeguamento alla realtà del tempo $»^{52}$. Attraverso i suoi epigoni, come Pietro d'Anzola ${ }^{53}$ e Pietro Boattieri, avrà la ventura di essere aggiornata ai tempi.

$E^{\prime}$ inutile qui sottolineare l'importanza di un simile filone letterario che, nei secoli, godrà di una fortuna non scarsa, attestata dalla grandissima mole di formulari redatti da più parti ${ }^{54}$ data l'innegabile importanza di una preparazione svolta anche su modelli idonei ad essere seguiti nella viva attività professionale. ${ }^{55}$

Non si tratta soltanto di formare il futuro notaio a fronte delle 'sfide' che la società comunale impone, dotandolo di una robusta preparazione tecnica, capace di affrontare i molteplici compiti e attività che lo sviluppo delle istituzioni impone, ma di renderlo idoneo e 'abilitato' a un inserimento via via più incisivo nel funzionamento dei nuovi organi.

Non tarda ad essere composto a Bologna, culla di una professione 'rigenerata' (il punto di partenza mi sembra quasi d'obbligo...) uno statuto del comune del 1251, che prescrive per il candidato, da sottoporre all'esame, che sia stato preliminarmente «in arte notarie adiscenda per unum annum»; nel 1257 il maestro, representator del candidato all'esame, deve garantire che costui «studuisse tribus annis in arte notarie» (a Bologna era già attiva da decenni, come si è accennato, una scuola di notariato, cresciuta all'ombra dello Studium felsineo e attenta alla cura del profilo teorico). Nel 1288 si prevede una preparazione propedeutica all'esame vero e proprio, con due anni di studio, per così dire di base, «in grammatica», e una preparazione specifica di un anno o più, «in documentis notarie sub ordinario doctore»; deve seguire un esame diligenter "de latino et hiis que spectant ad artem notarie». Negli statuti corporativi dei primi anni del Trecento, nel 1304, il regime diviene ancora più stringente, attraverso l'incremento, da due a quattro, degli studi in "grammatica», da uno a due, della pratica notarile. Una commissione, composta, con criteri selettivi, di sedici notai, quattro per quartiere "de melioribus et pericioribus et literatoribus», di un giudice del podestà e di un altro da lui nominato, è chiamata a giudicare «volentes fieri tabelliones

\footnotetext{
${ }^{50}$ Cfr. Bertram, 2002; Dolezalek, 2002; Pérez Martín, 2002

${ }^{51}$ Marmocchi, 2002.

52 di Renzo Villata, 2002a, spec. pp. 379-380.

${ }^{53} \mathrm{~V}$. i saggi raccolti in Tamba (ed.), 2014, in part. Giansante, 2014.

${ }^{54}$ Cfr. ad es. Sinisi, 2002.

${ }^{55}$ Cfr. Mangini, 2004.
} 
et inquirere diligenter ab eis de multis et diversis contractibus et videre et scire qualiter sciunt scribere et qualiter legere scripturas quas fecerint vulgariter et litteraliter et qualiter latinare et dictare». Nel 1336, in linea con le disposizioni del 1304, si penalizza il notaio inliteratus et indoctus, al punto tale che, in assenza di una "sufficentem litteraturam ad tabellionatus artem et officium exercendum», si prevede la cancellazione dalla matricola "et ulterius pro socio non habeatur» ${ }^{56}$. A Genova gli statuti corporativi del 1462, definiti da Lorenzo Sinisi «la charta magna del notariato genovese fino a tutto il Cinquecento", prescrivevano un iter complesso per accedere alla professione, requisiti, preesami, anche segreti ( «loco remoto») ed esami che dovevano verificare la preparazione «super gramaticalibus et arte notarie». Nonostante la serietà evidente nella normativa è stato rilevato che vi fosse una certa carenza di cultura giuridica, non superabile pur con i mezzi previsti in astratto per colmarla ${ }^{57}$

\section{II notaio e una moltitudine di competenze e attività: dal privato al pubblico}

Già si è qui fatta menzione, con rapidi cenni, dell'ampio ventaglio di attività via via svolte dal notaio nei secoli medievali e anche oltre ${ }^{58}$.

Gli esempi sono numerosi: basti scorgere gli statuti dell'età medievale per imbatterci nella figura del notaio che redige il testo assicurando anche una tendenziale uniformità almeno di linguaggio giuridico, se non di contenuti, nel segno della certezza del diritto: ${ }^{59}$ gli statuti di Grosseto, che documentano peraltro una frequentissima citazione dei notai impegnati presso i vari uffici del comune, deputati ad esercitare la molteplicità di funzioni essenziale per la vita regolare dell'istituzione, furono redatti in latino, nel 1421, dal notaio Naddo di Pietro di Naddo di Montalcino e, nel volgarizzamento in italiano, nel 1422, dal notaio Pietro di Giovanni di Cecchino di Montalcino («Questo statuto fu volgariçato per me Pietro di Giovanni Cecchini di Montalcino notaio cittadino di Siena nela terra di Montalcino et compiuto a dì ultimo di maggio .mccccxii. indictione .xv.»). Gli statuti di Montorsaio e la storia stessa di Montorsaio rivelano quanto il notaio fosse parte integrante del vissuto di una comunità soggetta a Siena ${ }^{60}$.

II Liber sententiarum potestatis Mediolani (1385), da poco edito, permette, scorrendolo, di imbattersi in una frequente clausola finale delle sentenze di questo tenore:

\footnotetext{
${ }^{56}$ Frati (ed.), 1869, p. 185-188; Fasoli- Sella, 1939, p. 49-50; Gaudenzi, 1896 (ed.), p. $40-$ 45; Sarti (ed.), 1988, spec. p. 47-48. V. Tamba, 1988; Tamba, 1998, spec. p. 314-315; da ultimo Tamba, 2009, spec. p. 207-208; Lombardo, 2012, spec. p. 263-264; già Ferrara, 1977. ${ }^{57}$ Sinisi, 1997, p. 167 ss., 172 ss.

${ }^{58}$ V., ad es. Tavilla, 2013, p. 143.

${ }^{59}$ Cfr. Piergiovanni, 2012, pp .375-380, spec. pp .378-379.

${ }^{60}$ Mordini (ed.),2019, spec. p. 296, ma, più specificamente Mordini, nella Premessa e nel cap. 3, rispettivamente p. XLVIII ss., ma anche passim; Mordini, 2004, passim.
} 
Ego Bernardus de Ghatis de Placentia, imperiali auctoritate notarius publicus, suprascriptas condemnationes pecuniarie et earum sententias mandato suprascripti domini potestatis scripsi, legi et publicavi ut supra et me subscripsi», "et lecte et publicate fuerunt suprascripte condempnationes et pecuniarie et absolutiones pecuniarie per me suprascriptum et infrascriptum Bartholomeum de Guinzonibus de Crema, notarium malleficiorum prefati domini potestatis et communis Mediolani, scriptis anno curente MCCCLXXXV $[\ldots]^{61}$.

Dell'uomo di cultura e politico basti qui citare un esempio 'celebrato': la figura di Coluccio Salutati, cancelliere della Repubblica fiorentina e non solo, ha suscitato l'interesse di una storiografia di varie competenze. E cancellieri notai se ne contano all'epoca, come il senza dubbio più modesto Benedetto Fortini... 62. Gli stessi manoscritti conservati dell'Ufficio dei Memoriali a Bologna, istituito nel 1265 e integrato, nella sua composizione, da un certo numero di notai, almeno quattro per semestre, impegnati ciascuno a scrivere ogni semestre un Liber memoralium, 'registrano' la presenza di rime di poeti bolognesi del Duecento, trascritte ad opera di notai come Enrichetto de Guerzi nel 1287 (Giosuè Carducci utilizzò la sua 'redazione' attribuendo il sonetto riportato Non mi potranno giammai fare ammenda a Dante Alighieri), ser Bonacosa di Giovanni nel 1279, Antonio di Guido da Argile nel 1282, Biagio di Auliverio e Niccolò di Filippo nel 1286, Niccolò di Giovannino Manelli nel 1287, Bonaccorso dei Rombolini e Filippo Bottrigari nel 1288, ser Venetico Aimeri nel 1294, Gerardo di Bonaventura nel 1309, Bonfigliuolo Zambeccari nel $1310^{63}$.

Quanto alle competenze in materia di miracoli, si tratta per il notaio di certificare un procedimento complesso e delicato che richiede l'ausilio del suo ruolo, capace di assicurare il sigillo della pubblica fede a atti suscettibili, anche a

${ }^{61}$ Pizzi (ed.), 2021, rispettivamente p. 46, 109, con riferimento alle clausole riportate supra nel testo, ma anche p. 109-110, 231-232, in ben sette sentenze del 5 agosto 1385, con lo stesso notaio Bartholomeus de Guinzonibus de Crema; pp. 116, 195, 227-228, 230 ( in due sentenze del 12 dicembre 1385), in cui il notaio è Franciscus de Melioratis de Crema «notarius imperiali auctoritate ac notarius malleficiorum preffati domini potesstatis $\mathrm{Me}$ diolani», come pure in due sentenze del $1^{\circ}$ luglio e quattro sentenze del 5 agosto 1385 ; p. 120 («Ego lohannis de Ottobellis de Alexandria, filius condam domini Petri, imperiali auctoritate notarius ac notarius malleficiorum prefati domini potestatis et communis Mediolani...») ancora Franceschino de Bordenaziis (pp. 228-229), Antonio de Montecornario (pp. 229-230), Giovanni de Orta (p. 240) e ad indicem. V. al riguardo i saggi raccolti in Bassani-Calleri-Mangini (edd.), 2021.

${ }^{62}$ La figura di Coluccio Salutati è stata al centro dell'attenzione di una storiografia internazionale, proveniente anche, in buona parte, dall'America: v. De Witt, 1983; in tempi recenti cfr. i saggi raccolti in Cardini e Viti, 2012, in part. Sznura, 2012; Quaglioni, 2012; Battista, 2012. Cfr., anche per un diverso ambiente territoriale, Zacchigna, 2018.

${ }^{63}$ Casini, 1981, p. XXIII e p. 174 , CV (del notaio Ser Antolino de' Rodaldi); p. XXII, e p. 35, XV (del notaio Ser Nicolò di Giovannino Manelli); p. XXIII e p. 149, XCII (del notaio Ser Venetico Aimeri). Carducci, 1876, p. 26. 
quei tempi, di generare, per così dire, dubbi: il suo 'impegno' è stato dimostrato da una storiografia volta ad indagare il coinvolgimento nella certificazione degli eventi soprannaturali in un'attività di 'autenticazione del sacro'. Tra i molti esempi che si potrebbero fare riguardo ai processi di canonizzazione svoltisi attraverso i secoli uno, ampiamente documentato per san Nicola da Tolentino, registra la presenza, come testimoni chiamati a deporre, di nove notai, nove iuris periti, tre doctores legum, con una preponderanza di un certo rilievo per il notaio di Tolentino Bernardus Appillattere. Si può rilevare come l'intervento dei notai parte attiva possa peraltro avvenire nella compilazione delle liste dei miracoli ${ }^{64}$.

\section{L'accentuarsi del controllo sull'attività notarile e gli archivi. Giambatti-} sta De Luca e i notai ne II dottor volgare (1673).... tra difetti e critiche ... verso la fine dell'ancien régime

Già si è accennato, quasi all'avvio di queste pagine, ad un declino delle fortune della categoria nel Cinquecento e al susseguirsi di elogi, difese, panegirici del ceto per rintuzzare le critiche e enfatizzare il ruolo indefettibile svolto.

Nella stessa epoca lo Stato prende ad interessarsi in maniera più penetrante dell'attività notarile: il potere pubblico ritiene di dovere incidere in funzione di garanzia di interessi diffusi e della fondamentale funzione svolta dalla categoria nel conferire pubblica fede agli atti rogati. Sono emanati molti provvedimenti per predisporre forme di controllo e di garanzia dell'attività notarile, qui solo esemplificati per individuare linee di tendenza.

Cosimo I, in Toscana, istituisce nel 1569 il Pubblico generale archivio dei contratti, operativo nel marzo 1570: con esso nasce l'obbligo di depositare tutte le scritture notarili, possedute a qualsiasi titolo dai privati o da istituzioni pubbliche, religiose o ecclesiastiche, ovvero magistrature o archivi, e rogate da notai defunti alla data del $1^{\circ}$ marzo 1570; altre regole sono fissate per disciplinare i rogiti. Nello Stato pontificio Sisto V promuove con la Solicitudo pastoralis officii (1588) la costituzione di centinaia di pubblici archivi notarili: a capo dell'assetto così creato è il Prefetto degli archivi; inoltre il papa si interessa vivamente alla professione e al suo ambito di competenze, soprattutto sul versante dei pubblici uffici dell'amministrazione ecclesiastica. Le Novae Constitutiones Mediolani (1541) hanno un titolo del libro II De publicis notariis, che contiene prescrizioni volte a disciplinare ed a sottoporre a controllo, sotto pena di sanzioni pecuniarie e accessorie della gravità della perdita dell'ufficio, i comportamenti dei notai pubblici, in particolare di quelli criminali, tali da concretizzare forme di abuso, di scorrettezza e di corruzione. Si ribadisce l'obbligo di registrazione degli atti di testamento, donazione e costituzione di dote a cura del notaio rogatario «penes officium gubernatoris statutorum communis Mediolani quod nuncupatur de Panigarolis». In Piemonte

\footnotetext{
${ }^{64} \mathrm{~V}$. i saggi raccolti in Michetti (ed.), 2004: in part. quello di Bartolomei Romagnoli, 2004; v. inoltre Occhioni (ed.), 1984.
} 
Carlo Emanuele I introduce nel 1610 il sistema dell' 'insinuazione', per il quale i notai sono tenuti a consegnare copia delle scritture e contratti, specificati dalla legge, a pubblici uffici, appositamente istituiti per conservarli in archivi a ciò destinati; nel 1679 si afferma la venalità dell'ufficio, che rende le piazze, predeterminate nel numero (tuttavia ridotto rispetto al passato), liberamente alienabili. Saranno poi le Regie Costituzioni del 1770 (titolo II del libro V) a dedicare al notariato molto rilievo. Il possesso di una piazza è il presupposto del rilascio della patente di approvazione e di esercizio del notariato: vi si accede all'età minima di venti anni, essendo di stato «laicale, di probità e buoni costumi, nato da onesti parenti e non esercenti un mestiere vile», in possesso di beni del valore di 4000 lire, o dando per la somma indicata una sufficiente cauzione, oltre all'obbligo di una pratica di tre anni presso l'ufficio di qualche causidico, di un esame teorico sulle "civili istituzioni», e di una prova pratica in materia contrattuale. A Modena, all'inizio del 1772, Francesco III istituisce una cattedra di notariato, unita al diritto patrio, nell'università appena riformata, mentre si prodiga per non lasciare più «gli istrumenti in sola e totale balia delli notari, che se ne rogarono, e dei loro eredi», o sparsi nei diversi archivi, mentre dispone di concentrare gli archivi in Modena, Reggio e Castelnuovo di Garfagnana, affidandone la gestione alla «custodia, governo e direzione» delle relative Congregazioni d'Archivio. Nella Lombardia austriaca già dal 1775 si realizza l'Archivio pubblico (Archivio notarile), di cui la documentazione dell'Archivio Panigarola, poi soppresso nel 1787, costituisce le fondamenta. Un Regolamento generale per i notari della Lombardia austriaca (1794) raccoglie e riassume una normativa sparsa sul notariato, fissa $\mathrm{i}$ requisiti per l'accesso (la nascita da legittimo matrimonio o la legittimazione per matrimonio susseguente, l'età minima di venticinque anni, la condizione di suddito per origine o per domicilio e lo stabile domicilio per dieci anni, il non avere esercitato "arte vile o ignobile nel comune concetto», l'avere il possesso attuale di un reddito annuo di lire 800 milanesi per lo Stato di Milano e di 1500 per lo Stato di Mantova, imponendo, tra l'altro, cosa non consueta nel panorama italiano di allora, la laurea o la licenza "dalla Regia Università di Pavia, o altrimenti» ${ }^{65}$. Si sono qui indicati solo alcuni - come si è prima accennato - dei tanti 'regolamenti' messi in atto per disciplinare e controllare.

II notaio continua comunque ad esercitare una funzione di grande rilievo, «tra privato e pubblico»: il ventaglio delle sue attribuzioni, nell'ambito statale ed ecclesiastico, nel privato, quale 'mediatore' di contrapposti interessi, non impedisce, non esclude che vi possa essere uno svolgimento della professione soddisfacente, al netto di un 'declino' forse lontano dal prestigio di un passato glorioso.

Nel maturo Seicento, l'utilità del notaio viene ribadita attraverso la voce di Giambattista De Luca, giurista-ecclesiastico, testimone attendibile della dottrina

\footnotetext{
${ }^{65}$ Per maggiori dettagli, per l'indicazione delle fonti e della bibliografia di riferimento, di Renzo Villata, 2009a, pp. 46-51; per la Lombardia Austriaca Salvi, 2012; Salvi, 2021 (in corso di stampa).
} 
e insieme della prassi coeva, destinato, pure con il suo porsi, in lingua volgare, ad una diffusione più generalizzata del suo pensiero: suggerisce di dare più peso ai requisiti sostanziali 'minimi' dell'atto notarile:

Sopra la fede degl'istromenti, e quando siano pubblici ed autentici, o no, ancorchè i giuristi vi formino gran questioni, e particolarmente coloro i quali camminano con la sola formalità della lettera delle leggi, cioè se il non esservi l'invocazione del nome di Dio, o del principe regnante, o l'indizione, vizi l'istromento e gli tolga la fede, tuttavia queste e simili considerazioni meritano di esser poste tra le solite freddure dei legisti. Attesochè ogni volta vi sia la podestà legittima del notaro, ed il numero sufficiente de' testimoni, con la certezza del luogo e del tempo, e delle persone cognite al notaro ed a' testimoni, in maniera che non vi sia un probabile sospetto della supposizione di una persona per l'altra; e per conseguenza che vi siano le cose sostanziali, dalle quali si renda certa la prova della verità per la fede pubblica, la quale per comun uso e per la libertà del commercio si deve dare al notaro, non conviene badare a queste sottigliezze, quando la legge particolare del luogo, o veramente lo stile, ordinato al medesimo fine di ovviare alle fraudi e di assicurarsi alla verità, non richiedesse diversamente, ordinando qualche formalità, la quale vi sia bisogno di osservare.

Di seguito, tiene in conto il particolarismo giuridico esasperato che ostacola «una regola certa e generale applicabile ad ogni caso e ad ogni luogo» nella fattispecie, ammettendo senza difficoltà l'efficacia di piena e concludente prova, «e conforme i giuristi dicono», di "prova provata» degli stessi strumenti. Né nasconde quanto la materia della fede da attribuirsi a tali atti prestasse il fianco a controversie e questioni, «e particolarmente quando non si giustificasse il notariato; ovvero che, questo giustificato, non fosse sufficiente, o che il carattere ed il sigillo del notaro non fossero bene riconosciuti».

Le espressioni usate sono chiaro indizio della diffidenza diffusa verso la categoria, che tuttavia merita di essere stimata per l'imprescindibile contributo alla certezza del diritto ${ }^{66}$.

In questa, necessariamente sommaria, carrellata sulla storia di una categoria, contraddistinta da alterne fortune, una menzione appare opportuna: si tratta del Decreto del 29 settembre-6 ottobre $1791^{67}$, negli anni della rivoluzione, non direttamente influente, nell'immediato, sulla nostra storia, ma all'origine di successivi sviluppi che invece peseranno. Composto nel segno di una denuncia degli abusi del passato, definisce I notai «des fonctionnaires publics chargés de recevoir tous les actes qui sont actuellement du ressort des notaires royaux et autres, et de leur donner le caractère d'authenticité attaché aux actes publics» (art. I, sez. II, tit.I). Vi sono nuove forme di nomina e di istituzione dei notai (tit. IV). Si fissano determinati requisiti di età ( 25 anni), di pratica professionale ( 8 anni) e di buona condotta.

\footnotetext{
${ }^{66}$ De Luca, 1840, pp. 396-397, ma v. anche De Luca, 1673, pp. 143-169.

${ }^{67}$ Duverger, 1824, pp. 462-471. V. Mazzanti Pepe, 1983, pp. 21-72.
} 


\section{II notaio nell'Ottocento italiano: dalla legge del 25 ventoso anno XI ai progetti di riforma tardo-ottocenteschi del Regno d'Italia... in dissolvenza}

La legge del 25 ventoso anno XI (16 marzo 1803) sul notariato, emanata in Francia, diventa il principale modello ispiratore degli ordinamenti del notariato negli stati italiani preunitari: l'art. 1 recita, riguardo alla funzione dei notai, qualificati come:

les fonctionnaires publics établis pour recevoir tous les acts et contrats auxquels les parties doivent ou veulent faire donner le caractère d'authenticité attaché aux actes de l'ìautorité publique, et pour en assurer la date, en conserver le dépôt, en délivrer des grosses et expèditions ${ }^{68}$.

La legge del Ventoso è divisa in tre titoli, i primi due divisi in sezioni, dedicati rispettivamente il primo alle funzioni e agli atti notarili, il secondo, assai corposo e qualificante, al numero dei notai, alla loro dislocazione territoriale e alla cauzione, alle condizioni di ammissione e al modo di nomina, oltre che alle Camere di disciplina, il terzo ai notai allora operativi: non sarà direttamente applicata nel Regno d'Italia, che ricomprende via via gran parte dell'Italia settentrionale ed alcuni Dipartimenti dell'Italia centrale.

Nel 1803, nei Piani di studi e di disciplina nelle Università nazionali, approntati per la riforma della pubblica istruzione nel Regno d'Italia, riconosciuta l'importanza, come strumento per documentare l'esistenza delle obbligazioni, della scrittura, eseguita "con certe forme», alcune prescritte dalla legge, altre confermate dalla consuetudine, altre «derivate dal leale e retto senso di chi è autorizzato ad estenderla, cioè del Notajo", si 'giustifica' l'urgenza di una conoscenza dei precetti dell'arte notarile, insegnati in maniera tale da garantire l'acquisizione di una specifica competenza a risolvere le questioni giuridiche, congiunta a quell' «incorruttibile integrità», considerata consustanziale al ruolo del notaio: "Perciò i precetti dell'arte notarile versar debbono nel far rilevare tra queste forme le più conducenti ad evitare le quistioni, onde al carattere $d^{\prime}$ incorruttibile integrità, che deve esser proprio del Notajo, sia congiunta la capacità di servirsi di quelle forme che assicurino i diritti delle obbligazioni e delle successioni» ${ }^{69}$.

Dal 1805 s'inizia una fase progettuale, affidata alla Commissione incaricata della redazione del nuovo Metodo giudiziario civile (poi accantonato per una rapida introduzione del Code de procédure civile): rispetto alla legge del Ventoso si avanzano soluzioni diversificate, talora abbandonate a favore di un adeguamento più forte al modello, talaltra conservate ed approvate. Mi piace ricordare, tra i requisiti per la nomina dei notai, I' " aver fatto in una delle Università del Regno il corso

${ }^{68}$ Bulletin des lois de la République française, 3 . $^{\text {e }}$ série, tome septième, 1803, p. 258. Per una traduzione v. Codice dei notari, 1808, pp. 5-31.

${ }^{69}$ Piani di Studi e di Disciplina per le Università Nazionali, in Foglio officiale della Repubblica Italiana, 1803, p. 174. 
degli studi prescritto dal piano di pubblica istruzione, e... aver riportato il corrispondente grado accademico» (un'eco dei trascorsi della Lombardia austriaca) , I' "aver fama di onestà e probità » (art. 14, n. 4 7), nonché l'esperimento di idoneità davanti alla Camera Notarile del Dipartimento a carattere pratico e teorico, con la redazione di un rogito, "che comprende due o più contratti, e un atto di ultima volontà», e un'interrogazione sull' «arte notariale».

Con la costituzione del Regno Lombardo-Veneto potrebbe sembrare quasi scontata l'adesione alla legge austriaca; invece si mantiene in vigore il regolamento per il notariato, introdotto nel Regno d'Italia durante il regime napoleonico, benchè, negli anni della restaurazione, si ponga a più riprese il problema "se abbia a continuarsi od abolirsi il notariato in queste province " ${ }^{70}$. L'ABGB, introdotto nelle province lombardo-venete con il 1816, lasciava libertà alle parti contrattuali di servirsi di scritture private, ma ciò non comporta conseguenze del tutto negative sul lavoro dei notai nel territorio perché, come rileverà qualche decennio dopo l'Avv. Daniele Lissoni, notaio in Milano, al momento dell'elaborazione, per impulso del Ministro Di Falco in epoca postunitaria di un progetto di legge per l'esercizio del notariato, nell'epoca precedente «i notai continuarono mai sempre ad essere richiesti, massime negli affari di maggiore importanza, non da altro più sostenuti che dalla pubblica fiducia $)^{71}$. Tuttavia la loro fama è in calo se Teresa Stampa, seconda moglie di Alessandro Manzoni, considererà sminuente per Tommaso Grossi: «... Lui, esser ridotto a far il Notajo! un Grossi! Mi vergogno per gl'Italiani in generale, e per i Milanesi in particolare! $\rangle^{72}$.

Negli ordinamenti preunitari della Restaurazione o si ritorna al precedente sistema, come avviene nel Regno di Sardegna, con l'editto 21 maggio 1814, o nel Ducato di Genova con qualche concessione alla tradizione del notariato genovese, o, come nel Ducato di Parma si mantengono le disposizioni di matrice francese, combinate con le tradizioni locali, espresse nella legge notarile dell' 8 gennaio 1821, reputata di alto livello, mentre, nello Stato pontificio, dopo un periodo di incertezza sui destini della categoria, un motu proprio del 22 agosto 1822 disciplina il notariato nell'intento di «rialzare all'antico splendore il ceto de' notai» e di uniformare il sistema archivistico introdotto secoli prima da Sisto $\mathrm{V}^{73}$.

Con l'unificazione del Regno d'Italia si succedono i progetti, le iniziative di associazioni notarili come l'Accademia notarile italiana o l'Associazione accademica dei notai d'Italia, di scarsa fortuna. Si discute molto sul volto che deve assumere la professione, sulla sua particolare posizione giuridica di pubblico funzionario e insieme esercente una libera professione. Una vivace azione propositiva è svolta proprio dal notariato del Nord Italia (sono sopratutto i notai parmensi, i modenesi, Elia Elia della Camera notarile milanese ad attivarsi), che mostra di avere e di

\footnotetext{
${ }^{70}$ Ancarani, 1983, p. 270 ss.

${ }^{71}$ Lissoni, 1868, p. 9.

72 L'Amico e Collega Prof. Gianmarco Gaspari mi ha fatto la segnalazione.

${ }^{73}$ Ancarani, 1983, pp. 317-334; di Renzo Villata, 2009a, pp. 55-61.
} 
volere proteggere interessi in parte contrapposti a quelli dei notai del Sud: si toccano profili delicati della professione poiché, a fronte di un «sistema della libertà", auspicato da Cavour in tutti i rami della pubblica amministrazione, sembrava preferirsi nei progetti uno "protezionista», operativo anche nel procedimento di nomina a vita, con decreto reale; si prevedono pubblici archivi, non approvati da diverse componenti della categoria; un'altra delicata questione è quella del titolo di studio necessario per accedere al notariato che, nel progetto del ' 68 , è indicato nella laurea in giurisprudenza, poi 'edulcorato' in un requisito meno rigoroso, come si vedrà.

Una tappa comunque significativa nello sviluppo di una legislazione influente in materia è la legge 25 luglio 1875 «riordinamento del notariato», accompagnata dal regolamento notarile 10 dicembre 1875 , a cui seguiranno nel '78/79 modifiche. Per essa i notai sono "uffiziali pubblici istituiti per ricevere gli atti tra vivi e di ultima volontà ed attribuire loro la pubblica fede, conservarne il deposito, rilasciarne le copie, i certificati e gli estratti». La legge regola la forma degli atti, della custodia degli stessi presso il notaio, della tenuta dei repertori, delle copie, degli atti rilasciati in originale, consigli e collegi notarili, archivi notarili, e prevede un'attività di vigilanza sui notai, sui consigli e sugli archivi. I requisiti per l'accesso alla professione ex art. 5 sono: 1) essere cittadino del Regno ed avere compiuto ventiquattro anni, o ventuno, se non vi siano altri concorrenti ventiquattrenni e il Consiglio notarile e la Corte d'appello formulino parere favorevole; 2) presentare i certificati di moralità; 3 ) aver compiuto nei modi stabiliti dalle leggi e dai regolamenti sulla pubblica istruzione i corsi delle istituzioni del diritto romano comparato col diritto patrio, dei codici civile e di procedura civile e del diritto commerciale (nel '79 anche diritto amministrativo) ed averne superati gli esami; 4) essere iscritto fra i praticanti presso un Consiglio notarile; 5) avere fatto la pratica, per due anni continui dopo l'iscrizione e gli esami sopra indicati, presso un notaio residente nel distretto del collegio; 6 ) avere sostenuto e superato un esame d'idoneità dopo avere compiuto la pratica notarile. Nonostante l'assenza della laurea tra i requisiti per l'accesso, molti notai l'avevano ugualmente conseguita $^{74}$. Nel 1879 il notaio lombardo Cattaneo ${ }^{75}$ poteva, dalle colonne del Monitore del notariato, e poi nel Giornale dei notari, nel 1881, sostenere la necessità di un titolo di studio adeguato:

È forse la professione notarile scesa così in basso dal posto onorifico che le accordava la sapienza romana, e che le tradizioni dell'Italia medioevale mantennero in fiore, da non essere stimata di concorrere in grado sociale con tanti impieghi, la maggiore essenziale importanza dei quali è discutibile, in quanto che per essi ci è modo di provvedere ad errori commessi dall'imperizia dei funzionari? I notai saranno, e dovranno essere, persone manuali, richieste solo a ricevere materialmen-

\footnotetext{
${ }^{74}$ Ancarani, 1983, pp. 349-464; Santoro, 1998, 39 ss., con analitica descrizione del dibattito e dei progetti.

${ }^{75}$ Cattaneo, 1879, p. 19.
} 
te gli atti? Perché non avranno quel solenne attestato della laurea che li costituisce in faccia al pubblico meglio capaci di estendere, colle cognizioni, coll'ordine e la chiarezza necessarii, gli instrumenti? A loro dovrà bastare una superficiale coltura [...]

La prima legge unitaria non soddisfa dunque la categoria che, in varie occasioni, nei congressi e sulla stampa specializzata continua a riflettere sui diversi aspetti e sulle possibilità di un suo miglioramento; progetti vengono avviati, ma occorrerà attendere fino al 1913 per una nuova disciplina ${ }^{76}$.

\section{II notaio nel Novecento}

La legge 16 febbraio 1913, n. 89 è il risultato di un lungo percorso che parte all'indomani dell'emanazione del testo unico del 1879. Congressi, articoli di notai su riviste specializzate alimentano il dibattito su snodi rimasti irrisolti, dall'associazionismo obbligatorio all'esclusività delle funzioni, all'esercizio di attività di giurisdizione volontaria, per indicare solo alcuni dei problemi di cui si discute.

La riforma, ispirata senza dubbio allo scopo di «elevare la condizione morale ed economica dei notai», accentua il loro profilo professionalizzante, ma... lascia alcuni dei problemi scottanti della categoria ancora in sospeso, come quello della concorrenza: nell'ibridismo del loro essere professionisti e pubblici ufficiali si cerca di far pendere la bilancia sul versante professionale.

Solo con la seconda metà del Novecento la professione vedrà in Italia una sua progressiva affermazione ed ascesa, anche grazie all'impulso dell'organo nazionale di categoria, il Consiglio Nazionale del Notariato.

Esercitando un ruolo di guida del ceto notarile, chiamato ad assumere un ruolo crescente nella vita sociale, si farà anche promotore di una serie di iniziative, di formazione, di aggiornamento, in supporto all'attività dei consociati, tra le quali emergono - mi sembra doveroso ricordarle qui -, per spessore qualitativo e quantitativo, quelle di taglio culturale.

\section{Un bilancio attraverso i secoli}

La professione, come spero di avere messo in luce nel corso di queste pagine, ha visto momenti di ascesa, soprattutto quelli medievali, seguiti da una fase meno, per così dire, spettacolare, contraddistinta dal crescere di controlli e da un calo di reputazione, protrattosi fino oltre le soglie dell'età contemporanea. Nel Novecento, dopo la legge del 1913, i notai hanno vissuto momenti di difficoltà e, con la caduta del regime, anni variamente soddisfacenti, in relazione pure alle migliori condizioni di vita ed economiche del Paese.

Gli anni del terzo millennio sembrano caratterizzati da una crisi delle vocazioni: sono quasi dimezzati i praticanti notai dal 2010 al 2020, mentre il reddito medio

\footnotetext{
${ }^{76}$ Santoro, 1998, passim.
} 
della categoria è sceso in una certa misura: si possono segnalare fattori di crisi dell'economia che, ripercuotendosi sulla minore mole di lavoro, non possono mancare di esercitare un ruolo in queste dinamiche. L'accesso delle donne si è di molto intensificato, pure se vi è tuttora una sproporzione tra la presenza femminile e quella maschile. Si discute - come è avvenuto nel LV congresso nazionale del notariato, svoltosi a inizio novembre 2021, degli effetti delle fake news sulla attendibilità delle informazioni e sul concetto di "publica fides", che ancora rimane una caratteristica intrinseca della funzione notarile. A partire dalla legge delega n. $110 / 2010$ l'attività del notaio ha subito importanti modifiche e si è accelerata la rivoluzione digitale: interrogatisi sui suoi effetti, pure si è affermato che il ruolo del notaio non ha subito alcun impoverimento visto che il suo compito «di indagare la volontà delle parti e di dirigere personalmente la compilazione integrale dell'atto, anzi - aggiungo io - assume una straordinaria densità tecnologica».

La dematerializzazione e l'informatizzazione, la formazione di un «ecosistema digitale in cui la disinformazione ha trovato il suo terreno fertile ${ }^{77}$ possono avere accelerato il processo involutivo? Al futuro la risposta, che potrà avere una connotazione positiva, nel segno di uno sviluppo e di una crescita consapevole, a mio modesto avviso, anche in rapporto alla capacità dei notai di 'reinventarsi' e di adeguarsi ai bisogni di una società in continuo movimento.

\section{Bibliografia}

Aliani A., 1995: Il notariato a Parma. La "Matricula Collegii Notariorum Parmae" (1406-1805), Milano, Giuffrè

Allingri M., 2018: L'activité des notaires siennois, fin XIIle-début XVe siècle: données prosopographiques et pistes d'interprétation, in G. Pinto, L. Tanzini, S. Tognetti (edd.), Notariorum itinera. Notai toscani del basso Medioevo tra routine, mobilità e specializzazione (Biblioteca storica toscana, LXXVIII), Firenze, Olschki, pp. 99-120

Amelotti M., Costamagna G., 1995: Alle origini del notariato italiano, Milano, Giuffrè

Angelini I., 2013: II notaio e poeta per passione Domenico Sacello, in "Archivio storico di Belluno Feltre e Cadore", 84/352 mag.-ag., pp. 95-108

Angelini I., 2014: Domenico Sacello: notaio e poeta nella Belluno del primo Cinquecento, in A. Giorgi, S. Moscadelli, G.M. Varanini, D. Quaglioni (edd.), II notariato nell'arco alpino. Produzione e conservazione delle carte notarili tra Medioevo ed Età Moderna, Convegno internazionale di Studi, Trento 24-26

\footnotetext{
77 Sono parole che si leggono online nella presentazione del Congresso del 4-5 novembre 2021 (https://www.notariato.it/it/news/55-congresso-nazionale-del-notariato-publica-fides-nel-mondo-delle-fake-news-roma-4-5-novembre-2021/).
} 
febbraio 2011 (Studi storici sul notariato italiano, XVI), Milano, Giuffrè, pp. 739752

Banti O., 1963/1964: Ricerche sul notariato a Pisa tra il secolo XIII e il secolo XIV, in "Bollettino Storico Pisano", XXXIII-XXXIV, pp. 131-186

Banti O., 1967: Ricerche sul notariato a Pisa tra il secolo XIII e il secolo XIV. Note in margine al Breve Collegii notariorum (1302), in Studi di storia pisana e toscana in onore del Prof. Ottorino Bertolini (Biblioteca del Bollettino storico pisano), Pisa-Livorno, Pacini, pp. 131-186

Banti O., 1989: II notaio e l'amministrazione del Comune a Pisa (secc. XII-XIV), in "Atti della Società Ligure di Storia Patria", n.s., XXIX/2, pp. 131-155

Barbagli A., 2011: Il notariato ad Arezzo tra Medioevo ed età Moderna (Quaderni di «Studi senesi»), Milano, Giuffrè

Baroni F.M, 1981: Novara e la sua diocesi nel Medio Evo attraverso le pergamene dell'Archivio di Stato, Novara, Banca Popolare

Baroni F.M, 1982: II documento notarile novarese: dalla "charta" all'"instrumentum", in "Studi di storia medioevale e diplomatica", 7, pp. 13-23

Bartoli Langeli A., 1992: Documentazione e notariato, in L. Cracco Ruggini, M. Pavan, G. Cracco, G. Ortalli, Storia di Venezia, I. Origini-Età Ducale, Roma, Istituto dell'Enciclopedia Italiana, pp. 847-864 (online: https://www.treccani. it/enciclopedia/eta-ducale-le-testimonianze-documentazione-e-notariato_ (Storia-di-Venezia)/)

Bartoli Langeli A, 2006: Notai: scrivere documenti nell'Italia medievale, Roma, Viella

Bartolomei Romagnoli A., 2004: Agiografi e notai. Due stili a confronto tra vite e processi di canonizzazione, in Notai miracoli e culto dei santi. Pubblicità e autenticazione del sacro tra XII e XV secolo, Milano, A. Giuffrè, pp. 203-231

Bassani A., Calleri M., Mangini M.L. (edd.), 2021: Liber sententiarum potestatis Mediolani (1385): Storia, diritto, diplomatica e quadri comparativi (Notariorum itinera), Genova, Società Ligure di Storia patria

Battista G., 2012: Benedetto Fortini successore di Salutati alla Cancelleria, in R. Cardini e P. Viti (edd.), Radici umanistiche dell'Europa: Coluccio Salutati cancelliere e politico. Atti del Convegno Internazionale del Comitato nazionale delle celebrazioni del VI centenario della morte di Coluccio Salutati FirenzePrato 9-12 dicembre 2008, (Humanistica 2/2), Firenze, Polistampa, pp. 171-195

Bertram M., 2002: I manoscritti delle opere di Rolandino conservati nelle biblioteche italiane e nella Biblioteca Vaticana, in G. Tamba (ed.), Rolandino e l'ars notaria da Bologna all'Europa. Atti del convegno internazionale di studi storici sulla figura e l'opera di Rolandino organizzato dal Consiglio notarile di Bologna sotto l'egida del Consiglio nazionale del notariato, Bologna, città europea della cultura, 9-10 ottobre 2000, Milano, Giuffrè, pp. 681-717 
Bettarini F., 2016: L'arte dei notai di Prato e lo statuto del 1332 (Notariorum itinera II), Genova, Società Ligure di Storia patria, Palazzo Ducale

Betto B., 1968: Uno statuto del collegio notarile di Treviso, in Contributi dell'Istituto di storia medioevale. Raccolta di studi in memoria di Giovanni Soranzo, Milano, Vita e Pensiero, pp. 10-59

Betto B., 1972: Strutture e compiti del Collegio notarle di Treviso attraverso documenti editi ed inediti, in Contributi dell'Istituto di storia medioevale, 2. Raccolta di studi in memoria di Sergio Mochi Honory, Milano, Vita e Pensiero, pp. 53-251

Betto B., 1981: I collegi dei notai, dei giudici, dei medici e dei nobili in Treviso (Secc. XIII-XVI): storia e documenti), Venezia, Deputazione di storia patria per le Venezie editrice

Birocchi I., 2013a: Rolandino Passeggeri (Passaggeri), (Bologna, 1215 ca.- ivi, ottobre 1300, in Dizionario Biografico dei Giuristi Italiani, pp. 1717-1720

Birocchi I., 2013b: Salatiele (1210/20-1280 ca.), in Dizionario Biografico dei Giuristi Italiani, pp. 1769-1771

Bruschi U., 2006: Nella fucina dei notai. L'Ars Notaria tra scienza e prassi a Bologna e in Romagna (fine XII - metà XIII secolo), Bologna, Bononia University Press

Boccaccio G., 2017: Decameron. Introduzione, note e repertorio di Cose e parole del mondo di A. Quondam, Testo critico e nota al testo a cura di M. Fiorilla, edizione rivista e aggiornata. Milano, BUR-Rizzoli

Calleri S., 1966: L'arte dei giudici e notai di Firenze nell'età comunale e nel suo Statuto del 1344, Milano, Giuffrè

Cammarosano P., 2001: Storia dell'Italia medievale. Dal VI all'XI secolo, RomaBari, Laterza

Cameli M. (a cura di), 2018: Padova 1419-1420. Gli statuti della fraglia dei notai (Padova, Biblioteca Civica, BP, 339), Roma, Istituto Storico Italiano per il Medio Evo

Cardini R. e Viti P. (edd.), 2012: Radici umanistiche dell'Europa: Coluccio Salutati cancelliere e politico. Atti del Convegno Internazionale del Comitato nazionale delle celebrazioni del VI centenario della morte di Coluccio Salutati FirenzePrato 9-12 dicembre 2008, (Humanistica 2/2), Firenze, Polistampa

Carducci G., 1876: Intorno ad alcune rime dei secoli XIII e XIV ritrovate nei memoriali dell'Archivio di Stato di Bologna, in Atti e Memorie della R. Deputazione di Storia Patria per le provincie di Romagna, Ser. II, vol. 11. Bologna, Romagnoli, p. 26

Cattaneo G., 1879: L'obbligo della laurea per i notai, in "Monitore del notariato", n. 2, pp. 17-20

Cattaneo G., 1881: Necessità della laurea in giurisprudenza per la nomina a notaio, in "Giornale de' notari", pp. 13-18 
Casini T., 1881: Le rime dei poeti bolognesi del secolo XIII, Bologna, Gaetano Romagnoli

Cencetti G., 1972: La "rogatio» nelle carte Bolognesi. Contributo allo studio del documento notarile italiano nei secoli X-X II, in Cencetti G., Notariato medievale Bolognese, pp. 217-352

Cencetti G., 1977: Notariato medievale bolognese (Scritti di G. Cencetti), Roma, Consiglio Nazionale del Notariato

Cheney C.R., Stein P.G., Brooks C.W., Helmholz R.H., 1972: Notaries Public in England in the Thirteenth and Fourteenth Centuries, Oxford, Oxford University Press

Cheney C. R., 1991: Notai in Inghilterra prima e dopo la riforma, Roma, Giuffrè

Chiodi G., 2002: Rolandino e il testamento, in G. Tamba (ed.), Rolandino e l'ars notaria da Bologna all'Europa. Atti del convegno internazionale di studi storici sulla figura e l'opera di Rolandino organizzato dal Consiglio notarile di Bologna sotto l'egida del Consiglio nazionale del notariato, Bologna, città europea della cultura, 9-10 ottobre 2000, Milano, Giuffrè, pp. 459-582

Clanchy, M.T., 1979: From Memory to Written Record, England 1066-1307, London, Edward Arnold

Codice dei notari promulgato in Francia li 25 ventoso anno XI,1808, Livorno, presso Tommaso Masi e Comp.

Condorelli O., 2009: Profili del notariato in Italia Meridionale, Sicilia e Sardegna (secoli XII-XIX), in M. Schmoeckel, W. Schubert, Handbuch zur Geschichte des Notariats der europäischen Traditionen, Baden-Baden, Nomosverlagsgesellschaft, pp. 65-124

Condorelli O., 2014: II significato del notariato per lo sviluppo della cultura giuridica europea (con particolare riferimento all'Italia), in P. Maffei e G.M. Varanini (edd.), Honos alit artes. Studi per il settantesimo compleanno di Mario Ascheri. Il cammino delle idee dal medioevo all'antico regime. Diritto e cultura nell'esperienza europea, Firenze, Firenze University Press, pp. 191-199

Costamagna G., 1970: II notaio a Genova tra prestigio e potere, Roma, Consiglio Nazionale del Notariato

De Lorenzi P., 1961-1962: Storia del notariato ravennate. I. L'organizzazione del notariato, II. L'evoluzione del sigillo, Ravenna, Arti grafiche

De Luca G.B., 1840: II dottor volgare, ovvero il compendio di tutta la legge civile, canonica, feudale e municipale nelle cose più ricevute in pratica, diviso in quattro volumi, Firenze, coi tipi di V. Batelli e c.

De Witt R., 1983: Hercules at the Crossroads: the Life, Works and Thought of Coluccio Salutati, Durham, Duke University Press

di Renzo Villata M.G., 2002a: // volto della famiglia medievale tra pratica e teoria 
nella Summa totius artis notariae, in G. Tamba (ed.), Rolandino e l'ars notaria da Bologna all'Europa. Atti del convegno internazionale di studi storici sulla figura e l'opera di Rolandino organizzato dal Consiglio notarile di Bologna sotto l'egida del Consiglio nazionale del notariato, Bologna, città europea della cultura, 9-10 ottobre 2000, Milano, Giuffrè, pp. 377-458

di Renzo Villata M.G., 2002b: Ludovico Antonio Muratori e la scienza giuridica della sua epoca tra conservazione e suggestioni di riforma, in Atti del Convegno. Giornata di Studi L.A. Muratori "I difetti della giurisprudenza ieri e oggi" (2 dicembre 2000), con il coordinamento di G. Alpa, Milano, pp. 83-119

di Renzo Villata M.G., 2009a: Per una storia del notariato nell'Italia centro-settentrionale, in Schmoeckel M., Schubert W., Handbuch zur Geschichte des Notariats der europäischen Traditionen, Baden-Baden, Nomosverlagsgesellschaft, pp. 15-64

di Renzo Villata M.G., 2009b: Ai margini della mostra. Tra la vita e la morte, tra passato, presente e futuro. Riflessioni sparse su testamenti, poesia, sentimenti e... interessi attraverso $i$ secoli, in $E$ viene il tempo della pietà: sentimento $e$ poesia nei testamenti, Catalogo della Mostra, 5 novembre 2009-26 febbraio 2010, Milano, pp. XLV-LXVII

di Renzo Villata M.G., 2016: II notariato nell'Italia del Sette-Ottocento tra cultura giuridica e pratica, in M.T. Guerrini, R. Lupi e M. Malatesta (edd.), Un monopolio imperfetto. Titoli di studio, professioni, università (secc. XIV-XXI) (CISUI. Studi 28), Bologna, CLUEB, pp. 131-152

Dolezalek G., 2002: La diffusione delle opere di Rolandino in Germania, in G. Tamba (ed.), Rolandino e l'ars notaria da Bologna all'Europa. Atti del convegno internazionale di studi storici sulla figura e l'opera di Rolandino organizzato dal Consiglio notarile di Bologna sotto l'egida del Consiglio nazionale del notariato, Bologna, città europea della cultura, 9-10 ottobre 2000, Milano, Giuffrè, pp. 737-757

Duverger J.-B., 1824: Décret sur la nouvelle organisation et sur le remboursement des offices des notaires, in Collection complète des lois, décrets, ordonnances, règlemens et avis du Conseil d'État..., tome III, Paris, chez a. Guyot et Scribe, pp. 462-471

Fasoli G., Sella P. (edd.), 1939: Statuti di Bologna del 1288, Città del Vaticano, Biblioteca Apostolica Vaticana

Ferrara R. (edd.), 1983: Rolandini Passagerii Contractus, Roma, Consiglio Nazionale del Notariato

Ferrara R., 1977: "Licentia exercendi» ed esame di notariato a Bologna nel secolo XIII, in Notariato medievale bolognese, II, Atti di un Convegno (febbraio 1976), Roma, Consiglio Nazionale del Notariato, pp. 47-120

Fissore G. G., 1977: Autonomia notarile e organizzazione cancelleresca nel 
comune di Asti (Biblioteca di studi medievali, IX), Spoleto, Fondazione CISAM Fissore G. G., 1998: Alle origini del documento comunale: i rapporti tra i notai e l'istituzione, in G. Albini (ed.) Le scritture del Comune: amministrazione e memoria nelle città dei secoli XII e XIII, Torino, Scriptorium, pp. 39-60

Foglio officiale della Repubblica Italiana, contenente i decreti, proclami, circolari ed avvisi riguardanti l'amministrazione pubblicati nel 1803, anno II, Milano, dalla Reale Stamperia, 1803

Frati L. (ed.), 1869: Statuti di Bologna dall'anno 1245 all'anno 1267, II, Bologna, Regia Tipografia

Gabotto F., Lizier A., Leone A., Morandi G. B., Scarzello O. (edd.), 1913: Le carte dell'Archivio Capitolare di S. Maria di Novara, I (729-1034) (BSSS,LX XV III), I, Novara, Tip. Parzini

Gaudenzi A., 1896: Statuti della società del popolo di Bologna, vol. II Società delle Arti, Roma, Forzani \& C. Tipografi del Senato

Gardoni G., 2013: Notai e Comuni nella Marca veronese: i protagonisti tra autonomia e subordinazione (secc. XII-XIII), in I. Lazzarini e G. Gardoni (edd.), Notariato e medievistica. Per i cento anni di studi e ricerche di diplomatica comunale di Pietro Torelli, Atti delle giornate di studi (Mantova, Accademia Nazionale Virgiliana, 2-3 dicembre 2011) Roma, ISIME - Istituto storico italiano per il Medioevo, pp. 261-287

Garzoni T., 1996: La piazza universale di tutte le professioni del mondo, a cura di B. Cherchi e P. Collina, Torino, Einaudi

Giansante M., 2000: I notai bolognesi tra cultura letteraria e impegno ideologico, in «I quaderni del M.AE.S.» 3, pp. 65-88

Giansante M., 2014: Pietro e i suoi maestri. Antichi e moderni nella storia del notariato bolognese, in G. Tamba (ed.), L'opera di Pietro d'Anzola per il notariato di diritto latino. Atti del Convegno di studi storici, Bologna-Anzola nell'Emilia 2012, Bologna, Forni Editore, pp. 101-122

Giansante M., 2017: Salatiele, in Dizionario Biografico degli Italiani, 89, Roma, Istituto dell'Enciclopedia Italiana, 2017, pp. 660-663

Gnavi A., 1990: Valori urbani e attività marginali nella Piazza universale di Tomaso Garzoni, in Ricerche storiche, XX, pp. 45-71

Grazia A. (a cura di), 1972: Salatiele, Summula de libellis, Bologna, Università degli Studi di Bologna, facoltà di Lettere e Filosofia (Studi e Ricerche, n.s. 26)

Grillo P., 2017: Repubbliche di notai? II ruolo politico del notariato nelle città italiane del secondo Duecento, in P. Grillo e S. Levati (edd.), Legittimazione e credito tra medioevo e Ottocento. Notai e ceto notarile tra ruoli pubblici e vita privata, Milano, FrancoAngeli, pp. 99-114

Lazzarini I. e Gardoni G. (edd.), 2013: Notariato e medievistica. Per i cento anni 
di studi e ricerche di diplomatica comunale di Pietro Torelli, Atti delle giornate di studi (Mantova, Accademia Nazionale Virgiliana, 2-3 dicembre 2011) Roma, ISIME - Istituto storico italiano per il Medioevo (anche online: www.biblioteca. retimedievali.it)

Leoni V., 2013: Notai e Comune a Cremona tra XII e XIII secolo. Note sui documenti pattizi tra il Comune cremonese e le città della regione padana (1183-1214), in I. Lazzarini e G. Gardoni (edd.), Notariato e medievistica. Per i cento anni di studi e ricerche di diplomatica comunale di Pietro Torelli, Atti delle giornate di studi (Mantova, Accademia Nazionale Virgiliana, 2-3 dicembre 2011) Roma, ISIME - Istituto storico italiano per il Medioevo, pp. 247-260

Lissoni D., 1868: Progetto di legge per l'esercizio del notariato, con annotazioni, cenni storici e raffronti, Milano, Tip. Boniardi-Pogliani di Ermen. Besozzi

Liva A., 1979: Notariato e documento notarile a Milano dall'Alto Medioevo alla fine del Settecento, Roma, Consiglio Nazionale del Notariato

Lombardo M. L., 2012: /l notaio romano tra sovranità pontificia e autonomia comunale (secoli XIV-XVI), Milano, Giuffrè

Magoni G., 1602: Lucernae moralis bipartite constituta cuius prima pars notariatus Auctoritatem ... nobile officium continet, secunda... notariatus fidelitatem indicat, Ticini, ex Typographia Petri Bartoli (online)

Mangini M. L., 2004: Infrascripta sunt necessaria sciri ad artem notarie. Un formulario notarile valtellinese della fine del XIV secolo, in "Archivio storico lombardo", 80, pp. 305-350

Marmocchi E., 2002: L'Aurora: Rolandino oltre l'Ars notaria, in G. Tamba (ed.), G. Tamba (ed.), Rolandino e l'ars notaria da Bologna all'Europa. Atti del convegno internazionale di studi storici sulla figura e l'opera di Rolandino organizzato dal Consiglio notarile di Bologna sotto l'egida del Consiglio nazionale del notariato, Bologna, città europea della cultura, 9-10 ottobre 2000, Milano, Giuffrè, pp. 667-679

Massetto G.P., 2002: Osservazioni in materia di contratti nella Summa totius artis notariae, in G. Tamba (ed.), Rolandino e l'ars notaria da Bologna all'Europa. Atti del convegno internazionale di studi storici sulla figura e l'opera di Rolandino organizzato dal Consiglio notarile di Bologna sotto l'egida del Consiglio nazionale del notariato, Bologna, città europea della cultura, 9-10 ottobre 2000, Milano, Giuffrè, pp. 249-328

Mazzanti Pepe F., Ancarani G., 1983: II notariato in Italia dall'età napoleonica all'unità (Studi storici sul notariato italiano VII), Roma, Consiglio Nazionale del Notariato

Meynial Ed., 1900/1901: Des renonciations au Moyen Âge et dans notre ancient droit, in "Nouvelle Revue historique de droit français et étranger", 24, pp. 108142 e 25 , pp. 241-277 
Michetti R. (a cura di), 2004: Notai miracoli e culto dei santi. Pubblicità e autenticazione del sacro tra XII e XV secolo (atti del Seminario internazionale, Roma, 5-7 dicembre 2002) (Studi storici sul notariato 12), Milano, A. Giuffrè

Montorzi M., 1984: Fides in rem publicam. Ambiguità e tecniche del diritto comune, Napoli, Jovene

Montorzi M., 1985: Il notaio di tribunale come pubblico funzionario: un primo quadro di problemi, e qualche spunto analitico (Studi storici sul notariato VIII), Roma, Giuffrè

Mordini M., 2004: La comunità di Montorsaio e i suoi statuti. Sviluppi storicoistituzionali dalla signoria rurale all'inserimento nello Stato di Siena, Grosseto, Biblioteca Comunale Chielliana di Grosseto- Amministrazione degli Usi Civici Montorsaio

Mordini M. (ed.), 2019: Statuta civitatis Grosseti (1421-1422), Siena, Accademia Senese degli Intronati

Muratori L.A., 1742: Dei difetti della giurisprudenza, Venezia, presso Giambattista Pasquali

Murray A.L., G. Dolezalek, 2020: Legal Argumentation and Citation of jus commune Sources in Pleadings in the Court of Session, 1549/1550, in A.M. Godfrey (ed.), Miscellany Eight (Stair Society 68), Edinburgh, pp. 113-230

Niccoli O., 1999: Garzoni Tomaso, in Dizionario Biografico degli Italiani, 52, pp. 449-453.

Occhioni N. (ed. crit.), 1984: Il processo per la canonizzazione di S. Nicola da Tolentino, Prefazione di A. Vauchez; Introduzione di D. Gentili, Tolentino, Padri Agostiniani di Tolentino- Roma, École française de Rome (Collection de l'École française de Rome 74)

Olivieri A., 2010: La società dei notai di Vercelli e i suoi statuti alla fine del Trecento, in A. Barbero e A. Comba (edd.), Vercelli nel secolo XIV. Atti del quinto Congresso storico vercellese (Biblioteca della Società Storica Vercellese), Vercelli, Saviolo edizioni. pp. 117-140

Olivieri A., 2013: Il salario del notaio ad officia. Spunti torelliani e ricerche regionali. Il caso di Torino nel Trecento e nei primi decenni del Quattrocento, in I. Lazzarini e G. Gardoni (edd.), Notariato e medievistica. Per i cento anni di studi e ricerche di diplomatica comunale di Pietro Torelli, Atti delle giornate di studi (Mantova, Accademia Nazionale Virgiliana, 2-3 dicembre 2011) Roma, ISIME - Istituto storico italiano per il Medioevo, pp. 213-230.

Orlandelli G., 1961: Salatiele, in II notariato nella civiltà italiana. Biografie notarili dall'VIII al XX secolo, Milano, Giuffrè, pp. 505-509

Orlandelli G., 1989: Documento e formulari bolognesi da Irnerio alla "Collecio contractuum di Rolandino, in J. Trenchs (éd.), Notariado público y documento privado: de los orígenes al siglo XIV, Generalitat Valenciana, Conselleria de 
Cultura, Educació | Ciència, Diputacions d'Alacant, Castelló | València

Padoa Schioppa A., 2002: Profili del processo civile nella Summa artis notariae di Rolandino, in G. Tamba (ed.), Rolandino e l'ars notaria da Bologna all'Europa. Atti del convegno internazionale di studi storici sulla figura e l'opera di Rolandino organizzato dal Consiglio notarile di Bologna sotto l'egida del Consiglio nazionale del notariato, Bologna, città europea della cultura, 9-10 ottobre 2000, Milano, Giuffrè, pp.583-610

Pagnin B., 1950: I/ documento privato veneziano, I. II formulario, Padova Crespano del Grappa, Tip. L. Mlchiori

Pedani Fabris M.P., 1996: "Veneta auctoritate notarius". Storia del notariato veneziano (1514-1797), Milano, Giuffrè

Pérez Martín A., 2002: La difusión de la obra de Rolandino en España, in G. Tamba (ed.), Rolandino e l'ars notaria da Bologna all'Europa. Atti del convegno internazionale di studi storici sulla figura e l'opera di Rolandino organizzato dal Consiglio notarile di Bologna sotto l'egida del Consiglio nazionale del notariato, Bologna, città europea della cultura, 9-10 ottobre 2000, Milano, Giuffrè, pp. 769-789

Pizzi F. (ed. crit.), 2021: Liber sententiarum potestatis Mediolani (1385), Genova, Società Ligure di Storia Patria

Preto P., 2020: Falsi e falsari nella storia: dal mondo antico a oggi, ed. W. Panciera e A. Savio, Roma, Viella

Puccinelli P., s.d. ma 1654/56 ca.: Della fede e nobiltà del notaio..., Milano, nella Regia Ducal Corte per Giulio Cesare Malatesta

Quaglioni D., 2012: "A problematical book»: il De tyranno di Coluccio Salutati, in R. Cardini e P. Viti (edd.), Radici umanistiche dell'Europa: Coluccio Salutati cancelliere e politico, pp. 335-350

Ramsay N., 2009: The History in England, in M. Schmoeckel, W. Schubert, Handbuch zur Geschichte des Notariats der europäischen Traditionen, BadenBaden, Nomosverlagsgesellschaft, pp. 375-391

Roccatagliata A. G., (1556): Ad Benedictum Ruscam Scriba, Genuae, Bellonus (anche online)

Roccatagliata A., 2002: La legislazione archivistica del comune di Alessandria, in "Rivista di storia, arte e archeologia per la provincia di Alessandria", CIX.2, pp. 391-421

Rolandino de' Passeggeri, 1546/1977: Summa totius artis notarie, Venetiis, apud luntas, 1546, rist. anast. Bologna, Forni

Rovere A., 2009: I lodi consolari e la documentazione pubblica nei più antichi cartolari notarili genovesi, in L. Paini (ed.), Studi in onore di Cesare Scalon, Udine (online: www.biblioteca.retimedievali.it). 
Rovere A., 2013: Comune e notariato a Genova: luci e ombre di un rapporto complesso, in I. Lazzarini e G. Gardoni G. (edd.), Notariato e medievistica. Per $i$ cento anni di studi e ricerche di diplomatica comunale di Pietro Torelli, Atti delle giornate di studi (Mantova, Accademia Nazionale Virgiliana, 2-3 dicembre 2011) Roma, ISIME - Istituto storico italiano per il Medioevo, pp. 231-245

Ruzzin V., 2015: Guglielmo (1191-1202): un notaio tra Genova Sori, in G. Oreste, D. Puncuh, V. Ruzzin (edd.), Guglielmo da Sori. Genova Sori e dintorni (1191,1195, 1201-1202), Genova, Società Ligure di Storia Patria (Notariorum Itinera I, 1), pp. XV-XLVIII

Ruzzin V. (ed.), 2018: "Notariorum itinera». Notai liguri del basso Medioevo tra routine, mobilità e specializzazioni, Genova, Società Ligure di Storia Patria (Notariorum Itinera Varia, 3)

Salatiele, 1584: Summula de libellis, in Tractatus Universi luris, III/2, Venetiis, [Società dell'Aquila che si rinnova]

Salvi S.T, 2012: Tra privato e pubblico. Notai e professione notarile a Milano (secolo XVIII), Milano, Giuffrè

Salvi S.T, 2021: Notai di età moderna mediatori? Qualche riflessione sul ruolo del notaio nella Lombardia austriaca (XVIII secolo), in corso di stampa

Santoro M., 1998: Notai. Storia sociale di una professione in Italia (1861-1940), Bologna, il Mulino

Sarti N. (ed.), 1988: Statuti della Società dei notai di Bologna dell'anno 1336. Contributo alla storia di una corporazione cittadina, Milano, Giuffrè

Schmidt T., 1988: Der ungetreue Notar, in Fälschungen im Mittelalter. Internationaler Kongre $\beta$ der Monumenta Germaniae Historica, München, 1619. September 1986, II, Hannover, Hahnsche Buchhandlung, pp. 691-711

Schmoeckel M., Schubert W., 2009: Handbuch zur Geschichte des Notariats der europäischen Traditionen, Baden-Baden, Nomosverlagsgesellschaft

Schmoeckel M., 2012: Die Reichsnotariatsordnung von 1512. Entstehung und Würdigung, in M. Schmoeckel, W. Schubert, Handbuch zur Geschichte des deutschen Notariats seit der Reichsnotariatsordnung von 1512, Baden Baden, Nomos (Rheinische Schriften zur Rechtsgeschichte 17), pp. 29-74

Sinisi L., 1997: Formulari e cultura giuridica notarile nell'età moderna. L'esperienza genovese, Milano, Giuffrè

Sinisi L., 2002: Alle origini del notariato latino: la Summa Rolandina come modello di formulario notarile, in G. Tamba (ed.), Rolandino e l'ars notaria da Bologna all'Europa. Atti del convegno internazionale di studi storici sulla figura e l'opera di Rolandino organizzato dal Consiglio notarile di Bologna sotto l'egida del Consiglio nazionale del notariato, Bologna, città europea della cultura, 9-10 ottobre 2000, Milano, Giuffrè, pp. 175-176

Sinisi L., 2006: "Judicis oculus». II notaio di tribunale nella dottrina e nella 
prassi di diritto comune», in V. Piergiovanni (ed.), Hinc publica fides. II notaio e l'amministrazione della giustizia. Atti del Convegno internazionale di studi storici, Genova 8-9 ottobre 2004, Milano, Giuffrè, pp. 217-240

Sinisi L., 2014: II notaio e la sua presenza nell'apparato giurisdizionale: profili storici, in I quaderni della Fondazione italiana del notariato e-library (https:// elibrary.fondazionenotariato.it/articolo.asp?art $=45 / 4512 \& \mathrm{mn}=3$ )

Sinisi L., 2020: Fra giurisdizione e documentazione. II notaio in tribunale dall'antico regime ai codici / Between Jurisdiction and Documentation. The Notary in the Court from the Ancien Régime to the Age of Modern Code, in "Jurisdictio. Storia e prospettive della Giustizia", n. 1-2020. Saggi 6 (https://www.iurisdictio.it/wpcontent/uploads/2020/08/N.-1-2020-S06.-SinisiOK.pdf)

Storti Storchi C., 2002: Compromesso e arbitrato nella Summa totius artis notariae di Rolandino, in G. Tamba (ed.), Rolandino e l'ars notaria da Bologna all'Europa. Atti del convegno internazionale di studi storici sulla figura e l'opera di Rolandino organizzato dal Consiglio notarile di Bologna sotto l'egida del Consiglio nazionale del notariato, Bologna, città europea della cultura, 9-10 ottobre 2000, Milano, Giuffrè, pp. 329-376

Sznura F., 1998: Per la storia del notariato fiorentino: i più antichi elenchi superstiti dei giudici e dei notai fiorentini (anni 1291 e 1338), in T. de Robertis e G. Savino (ed.), Tra libri e carte. Studi in onore di Luciana Mosiici, Firenze, F. Cesati editore, pp. 437-515

Sznura F., 2012: Appunti su Coluccio Salutati notaio e sul notariato fiorentino, in R. Cardini e P. Viti (edd.), Radici umanistiche dell'Europa: Coluccio Salutati cancelliere e politico, pp. 47-71

Tamba G., 1988: La società dei notai di Bologna, Roma, Ministero per i beni culturali e ambientali. Pubblicazioni degli Archivi di Stato. Strumenti CIII

Tamba G., 1998: Una corporazione per il potere. II notariato a Bologna in età comunale, Bologna, CLUEB

Tamba G. (ed.), 2002: Rolandino e l'ars notaria da Bologna all'Europa. Atti del Convegno internazionale di studi storici sulla figura e l'opera di Rolandino (Bologna, 9-10 ottobre 2000), Milano, Giuffrè

Tamba G., 2009: Da forza di governo a burocrazia. La trasformazione dei notai a Bologna nel secolo XIV, in V. Piergiovanni (ed.), II notaio e la città, Milano, Giuffrè, pp. 207-208

Tamba G. (ed.), 2014: L'opera di Pietro d'Anzola per il notariato di diritto latino. Atti del Convegno di studi storici, Bologna-Anzola dell'Emilia, 6 ottobre 2012, Sala Bolognese, Arnaldo Forni editore

Tarantino D., 2010: La fides instrumentorum. Per una storia del valore probatorio dei documenti nei processi civile e canonico dal tardo diritto comune ai codici, Fondazione Sergio Mochi Onory, Roma 
Tavilla E., 2013: Da notaio di città a notaio di Stato: la normativa nel Ducato estense (secc. XVIII-XIX), in G. Tamba, E. Tavilla, Nella città e per la città. I notai a Modena dal IX al XX secolo, Milano, Giuffrè, pp. 143-180

Tirelli V., 1985: II notariato a Lucca in epoca basso-medievale, In II notariato nella civiltà toscana (Studi storici sul notariato italiano 8), Roma, Consiglio Nazionale del Notariato

Varanini G.M., 2006: L'invenzione dei confini. Falsificazioni documentarie e identità comunitaria nella montagna veneta alla fine del medioevo e agli inizi dell'era moderna, in P. Guglielmotti (ed.), Distinguere, separare condividere Confini nelle campagne dell'Italia medievale, in "Reti medievali", VII, 1, pp. 1-27

Vitale V., 1949: Vita e commercio nei notai genovesi dei secoli XII e XIII, p. I, La vita civile (Atti della Società Ligure di storia patria, vol. LXXII, fasc. 1), Genova, Società ligure di storia patria

Zacchigna M., 2018: Notai, cancellieri e ceto politico nell'Italia nord-orientale fra Due e Quattrocento, Trieste, CERM 